\title{
Error Estimation for PGD Solutions: Dual Analysis and Adaptivity for Quantities of Interest
}

\author{
Jonatha Reis ${ }^{1,2}$, J. P. Moitinho de Almeida*1, Pedro Díez ${ }^{2,3}$, Sergio Zlotnik ${ }^{2,3}$
}

\begin{abstract}
When designing a structure or engineering a component, it is crucial to use methods that provide fast and reliable solutions, so that a large number of design options can be assessed. In this context, the Proper Generalized Decomposition can be a powerful tool, as it provides solutions to parametric problems, without being affected by the "curse of dimensionality".

Assessing the accuracy of the solutions obtained with the PGD is still a relevant challenge, particularly when seeking quantities of interest with guaranteed bounds.

In this work, we compute compatible and equilibrated PGD solutions and use them in a dual analysis to obtain quantities of interest and their bounds, which are guaranteed.

We also use these complementary solutions to compute an error indicator, which is used to drive a mesh adaptivity process, oriented for a quantity of interest. The corresponding solutions have errors that are much lower than those obtained using a uniform refinement or an indicator based on the global error, as the proposed approach focuses on minimizing the error in the quantity of interest.
\end{abstract}

Keywords: quantity of interest, error bounds, error estimation, Proper Generalized Decomposition, equilibrium formulation

Reis, J, Moitinho de Almeida, JP, Díez, P, Zlotnik, S. Error estimation for proper generalized decomposition solutions: Dual analysis and adaptivity for quantities of interest. Int J Numer Methods Eng. 2021; 122: 752- 776. https://doi.org/10.1002/nme.6559

\section{Introduction}

The current challenges in computational mechanics pertain to analyzing and handling large amounts of data, exploring high-dimensional parametric spaces and providing answers in short or real-time. This alludes to concepts like digital twins and technologies like machine learning, which are not only buzzwords, but methodologies to be considered in combination with the classical computational models $[7,11]$. Overall, an eye is to be kept out for the accuracy of the obtained numerical solutions, that has to be sufficient to make educated decisions, and not to be compromised by further approximations. Reduced Order Models (ROM) contribute to face these challenges by reducing the degrees of freedom of the models, suppressing redundancies in the description of the system to be modelled and simplifying the representation of the mathematical objects quantifying the physical magnitudes [17]. Among the standard ROM techniques, it is worth mentioning the Proper Orthogonal Decomposition (POD), that consists in building a reduced basis from a family of previously computed snapshots. In that sense, POD is classified as an a posteriori method, because it requires a prior and arbitrary sampling of the parametric space. POD is intimately related with machine learning, and it is actually a reinterpretation of Principal Component Analysis (PCA) to select the relevant modes,

\footnotetext{
${ }^{1}$ Department of Civil Engineering, Architecture and Georesources, Instituto Superior Técnico, University of Lisbon, Portugal

${ }^{2}$ Laboratori de Calcul Numeric (LaCaN), E.T.S. de Ingenieria de Caminos, Canales y Puertos, Universitat Politecnica de Catalunya, Spain

${ }^{3}$ International Centre for Numerical Methods in Engineering, CIMNE, Barcelona, Spain

*Corresponding author: J. P. Moitinho de Almeida. moitinho@civil.ist.utl.pt
} 
essential to describe the features of the system [30]. Alternatively to POD, the Proper Generalized Decomposition (PGD), is classified as an a priori strategy because the method itself provides the modes, with no need of producing a prior family of representative snapshots $[10,8]$. Moreover, PGD provides an explicit expression, a priori computed, of the parametric solution (the parametric dependence is explicit in the solution) and it is therefore well suited to provide real-time responses, which is a great advantage in the framework of digital twins [16].

The solutions obtained through reduced order methods are approximations of the real values, requiring verification and validation tools to attest their accuracy. There are several ways to analyze the errors in the approximations obtained, with early works for the error estimation of PGD approximations done by Ammar and colleagues [9]. Although these are valid forms of determining the errors of the approximations, the only way of obtaining solutions with guaranteed error bounds is by applying dual error analysis [13]. The idea behind the dual analysis is using a pair of complementary solutions for a specified problem and to compute bounds of errors for these solutions. The initial works using a combination of equilibrium and compatibility solutions to obtain bounds are associated with Prager and Synge [28], proceeded by the works from Fraeijs de Veubeke in the finite element method, with formulations of equilibrium elements [22]. Early works applying dual error analysis for PGD solutions were presented by Ladevèze and Chamoin, focusing in the constitute relation error and obtaining equilibrated fields from recovered solutions [26]. There are recent applications of this technique, namely the work by Chamoin and Thai, exploring isogeometric analysis and PGD solutions to deal with geometric parameters and applying the constitute relation error as an error estimator and indicator for mesh refinement $[2,12,14,15]$. Another approach using the dual error analysis concept was performed by Moitinho, using the extended formulation from Prager to general problems proposed by Debongnie and using a independent PGD solution, based on hybrid equilibrium finite elements, to define the equilibrated stress field [19, 3, 4, 18].

When analysing the errors of a solutions, it is often desirable to have information of specific quantities of interest (QoI). There are several works that takes the concept of goal-oriented formulations and apply it to reduced order methods, many specific for the PGD, but few that explore the use of the dual analysis in this context [21, 1, 25, 24].The solutions obtained from dual analysis are ideal when working with QoI, as they can provide high quality bounds of error on the QoI of the worst solution [5]. Dual analysis is also effective to drive adaptivity mesh refinement processes, as it provides information of the errors in the elements either in a global or in a local framework [6].

This paper focuses on the use of dual analysis to obtained bounds for QoI and to drive mesh adaptivity processes driven by global and local output errors in a PGD framework, assuming that the problem is exactly described by the equations considered. We briefly explain how to work with complementary solutions and dual analysis, detailing how to obtain local outputs which are average displacements at specific regions. The examples presented are solved using PGD approximations obtained using the algebraicPGDtools toolbox [20]. The solutions obtained are separated outputs that can be easily manipulated and compressed. We detail how to use the separated outputs to compute the error indicators, which are used in the academic problem that is studied.

\section{Governing Equations}

\subsection{Continuous Problem}

We consider a linear elastic body subjected to displacements $\mathbf{u}$ and defined in a Cartesian domain $\Omega \subset \mathbb{R}^{2}$. The boundary $\Gamma$ is decomposed in Neumann, $\Gamma_{N}$, and Dirichlet, $\Gamma_{D}$, conditions, such that $\Gamma_{N} \cap \Gamma_{D}=\emptyset$. The input loads are the body forces $\overline{\mathbf{b}}$, imposed tractions $\overline{\mathbf{t}}$, and prescribed boundary displacements $\overline{\mathbf{u}}$.

For our problem, the strain $\varepsilon$ can be obtained in Voigt's notation as:

$$
\varepsilon=\mathbf{D u},
$$

with the differential operator $\mathbf{D}$ defined as:

$$
\mathbf{D}=\left[\begin{array}{cc}
\frac{\partial}{\partial x} & 0 \\
0 & \frac{\partial}{\partial y} \\
\frac{\partial}{\partial y} & \frac{\partial}{\partial x}
\end{array}\right]
$$

Taking $\sigma$ as the Cauchy stress tensor, also represented in Voigt's notation, we can write the equilibrium equation as:

$$
\mathbf{D}^{T} \sigma+\overline{\mathbf{b}}=0
$$


The Dirichhlet boundary condition is defined as:

$$
\mathbf{u}-\overline{\mathbf{u}}=0 \quad \text { on } \Gamma_{D},
$$

and the Neumann boundary as:

$$
\text { n } \sigma-\overline{\mathbf{t}}=0 \quad \text { on } \Gamma_{N},
$$

with $\mathbf{n}$ being the boundary operator defined as:

$$
\mathbf{n}=\left[\begin{array}{ccc}
n_{x} & 0 & n_{y} \\
0 & n_{y} & n_{x}
\end{array}\right]
$$

Finally, considering $\mathcal{C}$ the elasticity operator, the constitutive relation reads:

$$
\varepsilon=\mathcal{C} \sigma
$$

\subsection{Compatible Finite Element Formulations}

When considering a compatible displacement field, we define the potential energy $\Pi$ of the system as the difference between the strain energy, $\mathcal{U}$, and the work of external forces, $\mathcal{V}$, such that:

$$
\Pi(\mathbf{u})=\mathcal{U}(\varepsilon(\mathbf{u}))-\mathcal{V}(\mathbf{u})=\frac{1}{2} \int_{\Omega}\left(\varepsilon(\mathbf{u})^{T} \mathcal{C}^{-1} \varepsilon(\mathbf{u})\right) \mathrm{d} \Omega-\int_{\Omega} \mathbf{u}^{T} \overline{\mathbf{b}} \mathrm{d} \Omega-\int_{\Gamma_{N}} \mathbf{u}^{T} \overline{\mathbf{t}} \mathrm{d} \Gamma
$$

We can obtain a solution from the set of all compatible displacement fields by minimizing the potential energy of the system. Considering a standard finite element approach, an approximate displacement field $\mathbf{u}_{h}$ can be defined as:

$$
\mathbf{u}_{h}=\mathbf{U} \hat{\mathbf{u}},
$$

where $\mathbf{U}$ is the matrix of displacement approximation functions and $\hat{\mathbf{u}}$ the vector of nodal unknowns. We can write the piecewise continuous associated strains in a similar way, such that

$$
\varepsilon_{h}=(\mathbf{D U}) \hat{\mathbf{u}}=\mathbf{B} \hat{\mathbf{u}} .
$$

Substituting the approximations (9) and (10) into Equation (8) and minimizing the resulting equation will lead to the following system:

$$
\mathcal{K} \hat{\mathbf{u}}=\hat{\mathbf{f}}
$$

which depends on the stiffness matrix $\mathcal{K}$, associated with the strain energy, and on the vector of equivalent generalised nodal forces $\hat{\mathbf{f}}$, associated with the work of the external forces. The approximated solutions (9) obtained from $\hat{\mathbf{u}}$ are generalised displacement fields that strongly satisfy the compatibility conditions and weakly satisfy equilibrium.

\subsection{Hybrid Equilibrium Finite Element Formulations}

The complementary potential energy $\Pi_{c}$, for an equilibrated Cauchy stress field $\sigma$, is the difference between the complementary strain energy, $\mathcal{U}_{c}$, and the work of the imposed displacements, $\mathcal{V}_{c}$, such that:

$$
\Pi_{c}(\sigma)=\mathcal{U}_{c}(\sigma)-\mathcal{V}_{c}(\sigma)=\frac{1}{2} \int_{\Omega}\left(\sigma^{T} \mathcal{C} \sigma\right) \mathrm{d} \Omega-\int_{\Gamma_{D}}(\mathbf{n} \sigma)^{T} \overline{\mathbf{u}} \mathrm{d} \Gamma .
$$

We seek a solution that minimizes this complementary potential energy $\Pi_{c}$. However, because of the tensorial nature of the stress field, its approximation is not as simple as it is for the displacements. This happens because interelement equilibrium is expressed in terms of the projection of the stress components on the interfaces, without requiring continuity of the components of the stress tensor. We choose to solve this problem by using a hybrid approach, noting that other alternatives are possible [23].

We begin by defining a hybrid energy $\mathcal{V}_{h}^{i}$ that projects the element tractions onto the displacements $\mathbf{v}$ of side $i$ of a given element, meaning that:

$$
\mathcal{V}_{h}^{i}(\mathbf{v}, \sigma)=\int_{\Gamma_{i}} \mathbf{v}^{T}(\mathbf{n} \sigma) \mathrm{d} \Gamma
$$


Then weak equilibrium on a side $i$ of an element belonging to $\Gamma_{N}$ is imposed by

$$
\mathcal{V}_{h}^{i}(\mathbf{v}, \sigma)=\mathcal{V}_{t}^{i}(\mathbf{v}) \quad \forall \mathbf{v}, \quad \text { with } \mathcal{V}_{t}^{i}(\mathbf{v})=\int_{\Gamma_{i}} \mathbf{v}^{T} \overline{\mathbf{t}} \mathrm{d} \Gamma
$$

Considering two generic elements $\mathrm{A}$ and $\mathrm{B}$ adjacent to the side $i$, we impose weak equilibrium by:

$$
\mathcal{V}_{h}^{i}\left(\mathbf{v}, \sigma_{A}\right)+\mathcal{V}_{h}^{i}\left(\mathbf{v}, \sigma_{B}\right)=0 \quad \forall \mathbf{v} .
$$

We approximate $\mathbf{v}$ as

$$
\mathbf{v}_{h}=\mathbf{V} \hat{\mathbf{v}} .
$$

These approximated displacements $\mathbf{v}_{h}$ correspond to the Lagrange multipliers of constraints (14) and (15).

The bases for the displacements on all the edges are assigned as columns of $\mathbf{V}$ and the weights defining their combination are represented in $\hat{\mathbf{v}}$.

The functions in $\mathbf{V}$ correspond to the weights that weakly impose equilibrium, guaranteeing an energetically consistent formulation. When the degree of the polynomial approximations is the same, verifying weak equilibrium implies that strong equilibrium is also satisfied.

We begin by expressing approximated Cauchy stresses $\sigma_{h}$ that are self-equilibrated stresses element-wise, without taking into account of what happens on their boundaries, so that:

$$
\sigma_{h}=\mathbf{S} \hat{\mathbf{s}},
$$

We can now combine Equation (12) with the constrains (14). Substituting the approximations (17) and (16) into (12) and (16) into (14), and minimizing the resulting energy leads to the following equations:

$$
\left[\begin{array}{cc}
-\mathcal{F} & \mathcal{D}^{T} \\
\mathcal{D} & \mathbf{0}
\end{array}\right]\left\{\begin{array}{c}
\hat{\mathbf{s}} \\
\hat{\mathbf{v}}
\end{array}\right\}=\left\{\begin{array}{c}
\hat{\mathbf{e}} \\
\hat{\mathbf{t}}
\end{array}\right\},
$$

which depend on the flexibility matrix $\mathcal{F}$, associated with the complementary strain energy, $\hat{\mathbf{e}}$, associated with the work of imposed displacements, and $\hat{\mathbf{t}}$, associated with the applied boundary tractions. The approximated solutions (17) obtained from $\hat{\mathbf{s}}$ defines the generalised stress field which strongly satisfy the equilibrium conditions. The approximations (16) obtained from $\hat{\mathbf{v}}$ define generalised boundary displacements which weakly satisfy compatibility.

\subsection{Projecting Complementary Solutions}

The projection of the equilibrated stresses $\sigma_{h}$ onto the compatible strains $\varepsilon_{h}$ is:

$$
\int_{\Omega} \sigma_{h}^{T} \varepsilon_{h} \mathrm{~d} \Omega=\hat{\mathbf{s}}^{T} \int_{\Omega} \mathbf{S}^{T} \mathbf{B} \mathrm{d} \Omega \hat{\mathbf{u}}=\hat{\mathbf{s}}^{T} \mathcal{S} \hat{\mathbf{u}}
$$

\section{Error Measures}

We seek to find approximated solutions of a physical problem, in which two error sources contribute to the difference between the physical response and the result provided by the numerical method. One associated with the proper description of the physical problem as a set of differential equations, and the other with the quality of the numerical solution of the said equations.

That being noted, in the following we will focus exclusively on the difference between the numerical solution and the solution of the differential equations, which are assumed to describe exactly the problem.

We use complementary solutions to compute local outputs and bounds of their errors. We present a detailed derivations of the equations used to obtain selected displacement averages, which is analogous to the equations presented in [5] to obtain stress averages. We briefly explain how to obtain bounds both in a goal-oriented and global framework, but the curious reader should proceed to [4] and references therein for more complete explanations. 


\subsection{Bound of the Global Errors}

Given a pair of dual solutions, we can compute a bound, $\epsilon^{2}$, of the square energy of their error as:

$$
\epsilon^{2}=\int_{\Omega}\left(\sigma_{k}-\sigma_{s}\right)\left(\varepsilon_{k}-\varepsilon_{s}\right) \mathrm{d} \Omega \geq\left\{\begin{array}{l}
\int_{\Omega}\left(\sigma-\sigma_{s}\right)\left(\varepsilon-\varepsilon_{s}\right) \mathrm{d} \Omega \\
\int_{\Omega}\left(\sigma_{k}-\sigma\right)\left(\varepsilon_{k}-\varepsilon\right) \mathrm{d} \Omega
\end{array}\right.
$$

where no subscript indicates the exact solution, the subscript $k$ indicates solutions that are kinematically admissible and $s$ solutions that are statically admissible. These relations apply to any pair of complementary approximations, which do not have to correspond to finite element solutions. In our case the PGD will be used.

Substituting the elasticity equations and assuming no initial strains, we rewrite Equation (20) element-wise, as a sum of $n_{e}$ elemental contributions:

$$
\epsilon^{2}=\sum_{e=1}^{n_{e}} \int_{\Omega_{[e]}}\left(\varepsilon_{k}^{T} \mathcal{C}^{-1} \varepsilon_{k}+\sigma_{s}^{T} \mathcal{C} \sigma_{s}-2 \sigma_{s}^{T} \varepsilon_{k}\right) \mathrm{d} \Omega=\sum_{e=1}^{n_{e}} \epsilon_{[e]}^{2} .
$$

We will often refer to the quantities obtained from this equation as the global error bounds, as they account for the error in the whole problem being studied.

\subsection{Local Outputs and their Bounds}

When solving an elasticity problem, it is common to have values that are more relevant for the design, such as stresses in zones with known concentrations or displacements that should not exceed a certain threshold. In order to look at these outputs we define local quantities, which represent weighted integrals of stresses or displacements in the zones of particular interest.

When these weighting functions are constant and equal to unity, the quantities will be average values of the fields multiplied by its dimension measure (volume, area or length). For example, when seeking the output along a line, the local quantity is the average value of the field at that line multiplied by its length. Different forms can be used for the weighting functions, with each form having a different physical meaning for the outputs obtained. For the examples described in this paper, we will always compute the local quantities as the average along lines.

We can compute the local quantities by applying the principle of virtual work, where the weighting functions act as virtual loads. In this text we will refer to these loads as virtual, in the sense that they do not need to exist. Note that these loads are also usually referred to in the literature as dual, adjoint or extractors. Likewise, the load that is physically applied to the structure is denoted as real.

From here on, a bar on top of a variable indicates that it is associated with the virtual problem, except for the prescribed actions.

Using stresses from the virtual solution $\bar{\sigma}_{s}$ that are equilibrated, we can obtain the exact local displacement output $\mathcal{L}_{k}(\mathbf{u})$ of the exact displacement field $\mathbf{u}$ as:

$$
\mathcal{L}_{k}(\mathbf{u})=\int_{\Omega} \bar{\sigma}_{s}^{T} \varepsilon \mathrm{d} \Omega-\int_{\Gamma_{D}}\left(\mathbf{n} \bar{\sigma}_{s}\right) \overline{\mathbf{u}} \mathrm{d} \Gamma
$$

and a similar expression is used to obtain an exact local stress output $\mathcal{L}_{s}(\sigma)[4]$.

Usually, it is not possible to obtain the exact solutions, hence we apply (22) to approximated solutions, in order to obtain estimates of the local output and bounds of their error.

To achieve this, we force the output derived from a compatible solution $\mathcal{L}_{k}\left(\mathbf{u}_{k}\right)$ to appear by adding and subtracting $\int_{\Omega} \bar{\sigma}_{s}^{T} \varepsilon_{k} \mathrm{~d} \Omega$ in Equation (22), so that:

$$
\mathcal{L}_{k}(\mathbf{u})=\int_{\Omega} \bar{\sigma}_{s}^{T}\left(\varepsilon-\varepsilon_{k}\right) \mathrm{d} \Omega+\underbrace{\int_{\Omega} \bar{\sigma}_{s}^{T} \varepsilon_{k} \mathrm{~d} \Omega-\int_{\Gamma_{D}}\left(\mathbf{n} \bar{\sigma}_{s}\right) \overline{\mathbf{u}} \mathrm{d} \Gamma}_{\mathcal{L}_{k}\left(\mathbf{u}_{k}\right)}
$$

where we introduce the local displacement output obtained from the compatible solution $\mathcal{L}_{k}\left(\mathbf{u}_{k}\right)$, noting that a similar expression can be obtained from the equilibrated solution which is denoted as $\mathcal{L}_{k}\left(\sigma_{s}\right)$. 
Now we manipulate Equation (23) to force a term that can be bounded to appear. We do so by adding and subtracting $\int_{\Omega} \bar{\sigma}_{k}^{T}\left(\varepsilon-\varepsilon_{k}\right) \mathrm{d} \Omega$ :

$$
\mathcal{L}_{k}(\mathbf{u})=\int_{\Omega}\left(\bar{\sigma}_{s}^{T}-\bar{\sigma}_{k}^{T}\right)\left(\varepsilon-\varepsilon_{k}\right) \mathrm{d} \Omega+\int_{\Omega} \bar{\sigma}_{k}^{T}\left(\varepsilon-\varepsilon_{k}\right) \mathrm{d} \Omega+\mathcal{L}_{k}\left(\mathbf{u}_{k}\right)
$$

The second integral in this expression is not computable without knowing the exact solution. By adding and subtracting $\int_{\Omega} \bar{\sigma}_{k}^{T}\left(\varepsilon-\varepsilon_{s}\right) \mathrm{d} \Omega$ in Equation (24) we define a corrected output $\tilde{\mathcal{L}}_{k}\left(\mathbf{u}_{k}\right)$, which has an error that is bounded, resulting in:

$$
\mathcal{L}_{k}(\mathbf{u})=\int_{\Omega}\left(\bar{\sigma}_{s}^{T}-\bar{\sigma}_{k}^{T}\right)\left(\varepsilon-\varepsilon_{k}\right) \mathrm{d} \Omega+\underbrace{\mathcal{L}_{k}\left(\mathbf{u}_{k}\right)+\int_{\Omega} \bar{\sigma}_{k}^{T}\left(\varepsilon_{s}-\varepsilon_{k}\right) \mathrm{d} \Omega}_{\tilde{\mathcal{L}}_{k}\left(\mathbf{u}_{k}\right)}+\int_{\Omega} \bar{\sigma}_{k}^{T}\left(\varepsilon-\varepsilon_{s}\right) \mathrm{d} \Omega
$$

Notice that the term $\int_{\Omega} \bar{\sigma}_{k}^{T}\left(\varepsilon-\varepsilon_{s}\right) \mathrm{d} \Omega$ is equivalent to $\int_{\Omega} \bar{\varepsilon}_{k}^{T}\left(\sigma-\sigma_{s}\right) \mathrm{d} \Omega$, which is zero because $\left(\sigma-\sigma_{s}\right)$ is a self balanced stress field and $\bar{\varepsilon}_{k}$ corresponds to a compatible displacement field with homogeneous boundary conditions. We can now rewrite Equation (25) to isolate the bounding term, so that:

$$
\mathcal{L}_{k}(\mathbf{u})-\tilde{\mathcal{L}}_{k}\left(\mathbf{u}_{k}\right)=\int_{\Omega}\left(\bar{\sigma}_{s}^{T}-\bar{\sigma}_{k}^{T}\right)\left(\varepsilon-\varepsilon_{k}\right) \mathrm{d} \Omega
$$

In an analogous way, by using $\sigma_{s}$ instead of $\mathbf{u}_{k}$ in Equation (24) we obtain an error associated with an equilibrated solution as:

$$
\mathcal{L}_{k}(\mathbf{u})-\mathcal{L}_{k}\left(\sigma_{s}\right)=\int_{\Omega}\left(\bar{\sigma}_{s}^{T}-\bar{\sigma}_{k}^{T}\right)\left(\varepsilon-\varepsilon_{s}\right) \mathrm{d} \Omega
$$

We can then average Equations (26) and (27) to write the error of the output associated with an averaged solution:

$$
\mathcal{L}_{k}(\mathbf{u})-\tilde{\mathcal{L}}_{k}\left(\sigma_{a}\right)=\int_{\Omega}\left(\bar{\sigma}_{s}^{T}-\bar{\sigma}_{k}^{T}\right)\left(\varepsilon-\varepsilon_{a}\right) \mathrm{d} \Omega
$$

Equation (28) is not computable because of its dependency on the exact value of $\varepsilon$, however we can apply the Cauchy-Schwarz inequality to it, which will lead to:

$$
\left|\mathcal{L}_{k}(\mathbf{u})-\tilde{\mathcal{L}}_{k}\left(\sigma_{a}\right)\right| \leq \underbrace{\sqrt{\int_{\Omega}\left(\bar{\sigma}_{s}^{T}-\bar{\sigma}_{k}^{T}\right)\left(\bar{\varepsilon}_{s}-\bar{\varepsilon}_{k}\right) \mathrm{d} \Omega}}_{\leq \bar{\epsilon}} \underbrace{\sqrt{\int_{\Omega}\left(\sigma^{T}-\sigma_{a}^{T}\right)\left(\varepsilon-\varepsilon_{a}\right) \mathrm{d} \Omega}}_{=\frac{1}{2} \epsilon} \leq \frac{1}{2} \bar{\epsilon} \epsilon=\epsilon_{a_{\text {local }}}
$$

This will result in an explicit definition of upper and lower bounds of the output:

$$
\tilde{\mathcal{L}}_{k}\left(\sigma_{a}\right)-\epsilon_{a_{\text {local }}} \leq \mathcal{L}_{k}(\mathbf{u}) \leq \tilde{\mathcal{L}}_{k}\left(\sigma_{a}\right)+\epsilon_{a_{\text {local }}} .
$$

Assuming there are no imposed displacements, we can substitute the elasticity equations to rewrite the corrected average local output $\tilde{\mathcal{L}}_{k}\left(\sigma_{a}\right)$ as:

$$
\tilde{\mathcal{L}}_{k}\left(\sigma_{a}\right)=\frac{1}{2} \int_{\Omega}\left(\bar{\sigma}_{s}^{T} \mathcal{C} \sigma_{s}+\bar{\sigma}_{s}^{T} \varepsilon_{k}-\bar{\varepsilon}_{k}^{T} \mathcal{C}^{-1} \varepsilon_{k}+\bar{\varepsilon}_{k}^{T} \sigma_{s}\right) \mathrm{d} \Omega
$$

We aim to compute the bounds as sums of elemental contributions, which may be used as mesh adaptivity indicators. One possible way of computing these contributions is using a modified version of the equations provided in [5]. We will assume that both compatible and equilibrated solutions have the same importance, which is the more general case. Hence, the square of the average local output error is:

$$
\bar{\epsilon} \epsilon=\epsilon_{a_{\text {local }}}^{2}=\frac{1}{8} \sum_{e}^{n_{e}}\left(\epsilon^{2} \bar{\epsilon}_{[e]}^{2}+\epsilon_{[e]}^{2} \bar{\epsilon}^{2}\right)
$$


We can also write a non squared version of the bounds, that comes from taking the bounds from a different set of equations [6]. The average local output error is then:

$$
\epsilon_{a_{\text {local }}}=\frac{1}{4} \sum_{e}^{n_{e}}\left(\bar{\epsilon}_{[e]}^{2} \frac{\epsilon}{\bar{\epsilon}}+\epsilon_{[e]}^{2} \frac{\bar{\epsilon}}{\epsilon}\right)
$$

The non squared version of the bounds is more suitable when paired with the local outputs, because of the consistency of the dimension of the bounds and the outputs. For mesh adaptivity purposes, the squared version can be better, as it gives more importance to the regions with higher values of the error indicator.

\section{Parametric Problem and PGD Approximations}

We want to obtain parametric solutions for our problem that depend on its material properties. We define a vector $\mu$, composed of $n_{p}$ parameters $\mu_{1}, \mu_{2}, \ldots, \mu_{n_{p}}$, where each parameter has its own one dimensional domain $\Omega_{i}$, with $i=1,2, \ldots, n_{p}$. The combined domain for vector $\mu$ is $\Omega_{\mu}=\Omega_{1} \otimes \Omega_{2} \otimes \cdots \otimes \Omega_{n_{p}}$, with $\mu \subset \Omega_{\mu}$.

Trying to find the solution to the continuous problem for all parameters described in $\mu$ is not feasible. Firstly, because it is not easy to find the exact solution for problems with complex geometries and secondly, because depending on the size of $\mu$, one will eventually run into the curse of dimensionality, where the cost to find the solution for all combinations of the parameters grows exponentially with every parameter added.

A solution for this issue is using reduced models. In our case we use PGD approximations, specifically the ones obtained using the algebraicPGDtools toolbox. These solutions have a separated tensor form, making them easier to manipulate. In each element we can write the PGD approximations of the parameters of the finite element solutions (nodal or modal), either for the displacements $\hat{\mathbf{u}}$ or for the stresses $\hat{\mathbf{s}}$, as follows:

$$
\begin{aligned}
{[\hat{\mathbf{u}}]_{e}(\mu) } & =\sum_{m=1}^{n_{k}}\left[\overline{\mathbf{u}}^{m}\right]_{e} \prod_{i=1}^{n_{p}} U_{i}^{m}\left(\mu_{i}\right) ; \\
{[\hat{\mathbf{s}}]_{e}(\mu) } & =\sum_{m=1}^{n_{s}}\left[\overline{\mathbf{s}}^{m}\right]_{e} \prod_{i=1}^{n_{p}} S_{i}^{m}\left(\mu_{i}\right),
\end{aligned}
$$

where we denote the spatial modes for the displacement vector as $\overline{\mathbf{u}}$ and for the stress as $\overline{\mathbf{s}}$. The nodal functions of each parameters are described using the matrices $U(\mu)$ and $S(\mu)$. One requirement of the algebraicPGDtools is that the input values must be provided in a separated manner. Hence, the separated form of the stiffness and flexibility matrices $\mathcal{K}$ and $\mathcal{F}$, for element $e$, can be written as:

$$
\begin{aligned}
& \mathcal{K}_{[e]}(\mu)=\sum_{m=1}^{n_{C}} \overline{\mathcal{K}}_{[e]}{ }^{m} \prod_{i=1}^{n_{p}} C_{i}^{m}\left(\mu_{i}\right) \\
& \mathcal{F}_{[e]}(\mu)=\sum_{m=1}^{n_{C}} \overline{\mathcal{F}}_{[e]}^{m} \prod_{i=1}^{n_{p}} C_{i}^{m}\left(\mu_{i}\right) .
\end{aligned}
$$

Notice that these separated forms also require the elasticity operator and its inverse in a separated form, which yields $\underset{\sim}{C}$ and $C$. Details of these computations are presented in [29].

Given the approximated solutions for the displacements and stresses, we can apply them to find the parametric form of the errors indicators.

\subsection{PGD Approximated Energy of the Error}

The parametric form of the bound of the energy of the error can be written as:

$$
\epsilon^{2}(\mu)=\sum_{e=1}^{n_{e}} \epsilon_{[e]}^{2}(\mu)=\sum_{e=1}^{n_{e}}\left([\hat{\mathbf{u}}]_{e}^{T}(\mu) \mathcal{K}_{[e]}(\mu)[\hat{\mathbf{u}}]_{e}(\mu)+[\hat{\mathbf{s}}]_{e}^{T}(\mu) \mathcal{F}_{[e]}(\mu)[\hat{\mathbf{s}}]_{e}(\mu)-2[\hat{\mathbf{s}}]_{e}^{T}(\mu) \mathcal{S}_{[e]}[\hat{\mathbf{u}}]_{e}(\mu)\right)
$$


Substituting (34), (35), (36) and (37) into (38), we have:

$$
\begin{aligned}
\epsilon^{2}(\mu)=\sum_{e=1}^{n_{e}} \epsilon_{[e]}^{2}(\mu)= & \sum_{e=1}^{n_{e}}\left(\left(\sum_{m=1}^{n_{k}}\left[\overline{\mathbf{u}}^{m}\right]_{e} \prod_{i=1}^{n_{p}} U_{i}^{m}\left(\mu_{i}\right)\right)^{T}\left(\sum_{c=1}^{n_{C}} \overline{\mathcal{K}}_{[e]}^{c} \prod_{i=1}^{n_{p}} C_{i}^{c}\left(\mu_{i}\right)\right)\left(\sum_{k=1}^{n_{k}}\left[\overline{\mathbf{u}}^{k}\right]_{e} \prod_{i=1}^{n_{p}} U_{i}^{k}\left(\mu_{i}\right)\right)+\right. \\
& \left(\sum_{m=1}^{n_{s}}\left[\overline{\mathbf{s}}^{m}\right]_{e} \prod_{i=1}^{n_{p}} S_{i}^{m}\left(\mu_{i}\right)\right)^{T}\left(\sum_{c=1}^{n_{C}} \overline{\mathcal{F}}_{[e]}^{c} \prod_{i=1}^{n_{p}} C_{i}^{c}\left(\mu_{i}\right)\right)\left(\sum_{k=1}^{n_{s}}\left[\overline{\mathbf{s}}^{k}\right]_{e} \prod_{i=1}^{n_{p}} S_{i}^{k}\left(\mu_{i}\right)\right)- \\
& \left.2\left(\sum_{m=1}^{n_{s}}\left[\overline{\mathbf{s}}^{m}\right]_{e} \prod_{i=1}^{n_{p}} S_{i}^{m}\left(\mu_{i}\right)\right)^{T} \overline{\mathcal{S}}_{[e]}\left(\sum_{k=1}^{n_{k}}\left[\overline{\mathbf{u}}^{k}\right]_{e} \prod_{i=1}^{n_{p}} U_{i}^{k}\left(\mu_{i}\right)\right)\right) ;
\end{aligned}
$$

so that,

$$
\begin{aligned}
\epsilon^{2}(\mu)= & \sum_{e=1}^{n_{e}} \epsilon_{[e]}^{2}(\mu)=\sum_{e=1}^{n_{e}}\left(\sum_{m=1}^{n_{k}} \sum_{k=1}^{n_{k}} \sum_{c=1}^{n_{C}}\left[\overline{\mathbf{u}}^{m^{T}}\right]_{e} \overline{\mathcal{K}}_{[e]}^{c}\left[\overline{\mathbf{u}}^{k}\right]_{e} \prod_{i=1}^{n_{p}} U_{i}^{m}\left(\mu_{i}\right) C_{i}^{c}\left(\mu_{i}\right) U_{i}^{k}\left(\mu_{i}\right)+\right. \\
& \left.\sum_{m=1}^{n_{s}} \sum_{k=1}^{n_{s}} \sum_{c=1}^{n_{C}}\left[\overline{\mathbf{s}}^{m^{T}}\right]_{e} \overline{\mathcal{F}}_{[e]}^{c}\left[\overline{\mathbf{s}}^{k}\right]_{e} \prod_{i=1}^{n_{p}} S_{i}^{m}\left(\mu_{i}\right) C_{i}^{c}\left(\mu_{i}\right) S_{i}^{k}\left(\mu_{i}\right)-2 \sum_{m=1}^{n_{s}} \sum_{k=1}^{n_{k}}\left[\overline{\mathbf{s}}^{m^{T}}\right]_{e} \mathcal{S}_{[e]}\left[\overline{\mathbf{u}}^{k}\right]_{e} \prod_{i=1}^{n_{p}} S_{i}^{m}\left(\mu_{i}\right) U_{i}^{k}\left(\mu_{i}\right)\right) .
\end{aligned}
$$

If we define:

$$
\begin{aligned}
& m_{K}=n_{k}^{2} n_{\mathcal{C}} \quad m_{F}=n_{s}^{2} n_{C} \quad m_{S}=n_{k} n_{s}
\end{aligned}
$$

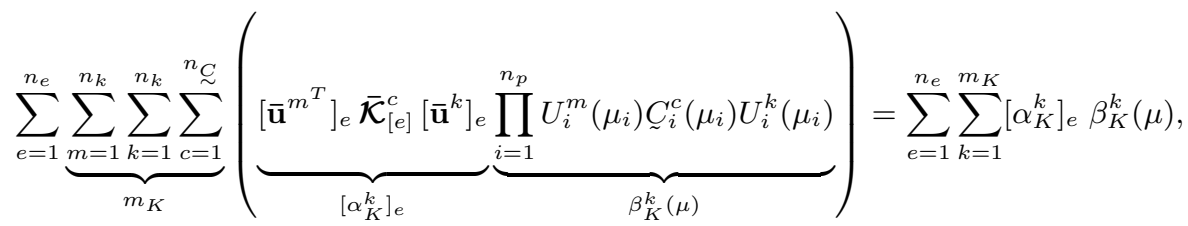

$$
\begin{aligned}
& \sum_{e=1}^{n_{e}} \underbrace{\sum_{m=1}^{n_{s}} \sum_{k=1}^{n_{s}} \sum_{c=1}^{n_{C}}}_{m_{F}}(\underbrace{\left[\overline{\mathbf{s}}^{m^{T}}\right]_{e} \overline{\mathcal{F}}_{[e]}^{c}\left[\overline{\mathbf{s}}^{k}\right]_{e}}_{\left[\alpha_{F}^{f}\right]_{e}} \underbrace{\prod_{i=1}^{n_{p}} S_{i}^{m}\left(\mu_{i}\right) C_{i}^{c}\left(\mu_{i}\right) S_{i}^{k}\left(\mu_{i}\right)}_{\beta_{F}^{f}(\mu)})=\sum_{e=1}^{n_{e}} \sum_{f=1}^{m_{F}}\left[\alpha_{F}^{f}\right]_{e} \beta_{F}^{f}(\mu), \\
& \sum_{e=1}^{n_{e}} \underbrace{\sum_{m=1}^{n_{s}} \sum_{k=1}^{n_{k}}}_{m_{S}}(\underbrace{\left[\overline{\mathbf{s}}^{m^{T}}\right]_{e} \overline{\mathcal{S}}_{[e]}^{c}\left[\overline{\mathbf{u}}^{k}\right]_{e}}_{\left[\alpha_{S}^{s}\right]_{e}} \underbrace{\prod_{i=1}^{n_{p}} S_{i}^{m}\left(\mu_{i}\right) U_{i}^{k}\left(\mu_{i}\right)}_{\beta_{S}^{s}(\mu)})=\sum_{e=1}^{n_{e}} \sum_{s=1}^{m_{S}}\left[\alpha_{S}^{s}\right]_{e} \beta_{S}^{s}(\mu),
\end{aligned}
$$

we can simplify Equation (40) to:

$$
\epsilon^{2}(\mu)=\sum_{e=1}^{n_{e}} \epsilon_{[e]}^{2}(\mu)=\sum_{e=1}^{n_{e}}\left(\sum_{k=1}^{m_{K}}\left[\alpha_{K}^{k}\right]_{e} \beta_{K}^{k}(\mu)+\sum_{f=1}^{m_{F}}\left[\alpha_{F}^{f}\right]_{e} \beta_{F}^{f}(\mu)-2 \sum_{s=1}^{m_{S}}\left[\alpha_{S}^{s}\right]_{e} \beta_{S}^{s}(\mu)\right) .
$$

Notice that the expression in (41) rearranges the equivalent expression in [29], merging sums and indices in order to produce a more compact form.

We also want to use the bound of the energy of the error to drive an adaptivity process, but (41) is not a good adaptivity indicator, as each set of parameters will lead to different values of the contributions to the bound. An 
alternative to this is to perform an integral of the bound of the energy of the error in the parametric space as well. This will lead to:

$$
\begin{aligned}
\int_{\Omega_{\mu}} \epsilon^{2}(\mu) \mathrm{d} \Omega_{\mu}= & \int_{\Omega_{\mu}} \sum_{e=1}^{n_{e}} \epsilon_{[e]}^{2}(\mu) \mathrm{d} \Omega_{\mu}= \\
& \sum_{e=1}^{n_{e}}\left(\sum_{k=1}^{m_{K}}\left[\alpha_{K}^{k}\right]_{e} \int_{\Omega_{\mu}}\left(\beta_{K}^{k}(\mu)\right) \mathrm{d} \Omega_{\mu}+\sum_{f=1}^{m_{F}}\left[\alpha_{F}^{f}\right]_{e} \int_{\Omega_{\mu}}\left(\beta_{F}^{f}(\mu)\right) \mathrm{d} \Omega_{\mu}-2 \sum_{s=1}^{m_{S}}\left[\alpha_{S}^{s}\right]_{e} \int_{\Omega_{\mu}}\left(\beta_{S}^{s}(\mu)\right) \mathrm{d} \Omega_{\mu}\right)
\end{aligned}
$$

For simplicity, we will define (42) as:

$$
\Psi_{\text {global }}^{2}=\int_{\Omega_{\mu}} \epsilon^{2}(\mu) \mathrm{d} \Omega_{\mu}
$$

\subsection{PGD Approximated Local Outputs}

After solving the integrals in Equation (31), we can write the parametric form of the approximate local outputs as:

$$
\tilde{\mathcal{L}}_{k}\left(\sigma_{a}(\mu)\right)=\frac{1}{2} \sum_{e=1}^{n_{e}}\left([\overline{\hat{s}}]_{e}^{T}(\mu) \mathcal{F}_{[e]}(\mu)[\hat{\mathbf{s}}]_{e}(\mu)+[\overline{\hat{s}}]_{e}^{T}(\mu) \mathcal{S}_{[e]}[\hat{\mathbf{u}}]_{e}(\mu)-[\overline{\hat{u}}]_{e}^{T}(\mu) \mathcal{K}_{[e]}(\mu)[\hat{\mathbf{u}}]_{e}(\mu)+[\overline{\hat{u}}]_{e}^{T}(\mu) \mathcal{S}_{[e]}^{T}[\hat{\mathbf{s}}]_{e}(\mu)\right) .
$$

For simplicity, we suppress the dependency of $\tilde{\mathcal{L}}_{k}$ on $\sigma_{a}$ and use $\tilde{\mathcal{L}}_{k}(\mu)$ instead. We can substitute Equations $(34)$, (35), (36) and (37) into Equation (44) and use an equivalent definition for the $\alpha$ and $\beta$ values, adding a tilde to indicate that these variables result from a combination of the real and virtual problems. The resulting equation is:

$$
\tilde{\mathcal{L}}_{k}(\mu)=\frac{1}{2} \sum_{e=1}^{n_{e}}\left(\sum_{\tilde{f}=1}^{\tilde{m}_{F}}\left[\tilde{\alpha}_{F}^{\tilde{f}}\right]_{e} \tilde{\beta}_{F}^{\tilde{f}}(\mu)+\sum_{\tilde{s}=1}^{\tilde{m}_{S}}\left[\tilde{\alpha}_{S}^{s}\right]_{e} \tilde{\beta}_{S}^{s}(\mu)-\sum_{\tilde{k}=1}^{\tilde{m}_{K}}\left[\tilde{\alpha}_{K}^{k}\right]_{e} \tilde{\beta}_{K}^{\tilde{k}}(\mu)+\sum_{\tilde{s}=1}^{\tilde{m}_{S}}\left[\tilde{\alpha}_{S^{T}}^{s}\right]_{e} \tilde{\beta}_{S^{T}}^{s}(\mu)\right) .
$$

Notice that we can write the elemental contributions of the approximated local outputs in a form that is similar to (41), however, since the value of the local output in a single element does not have a physical meaning and will not be used in our computations, the corresponding equation is omitted.

\subsection{PGD Approximated Bounds of the Local Output Error}

If we consider that $\epsilon^{2}$ comes from the real problem, we can define a similar $\bar{\epsilon}^{2}$ for the virtual problem. That is:

$$
\bar{\epsilon}^{2}(\mu)=\sum_{e=1}^{n_{e}} \bar{\epsilon}_{[e]}^{2}(\mu)=\sum_{e=1}^{n_{e}}\left(\sum_{\bar{k}=1}^{\bar{m}_{K}}\left[\bar{\alpha}_{K}^{\bar{k}}\right]_{e} \bar{\beta}_{K}^{\bar{k}}(\mu)+\sum_{\bar{f}=1}^{\bar{m}_{F}}\left[\bar{\alpha}_{F}^{\bar{f}}\right]_{e} \bar{\beta}_{F}^{\bar{f}}(\mu)-2 \sum_{s=1}^{\bar{m}_{S}}\left[\bar{\alpha}_{S}^{\bar{s}}\right]_{e} \bar{\beta}_{S}^{\bar{s}}(\mu)\right)
$$

We can write the parametric version of the square of the bound of the local output error of the average solution, from Equation (32), as:

$$
\epsilon_{a_{\text {local }}}^{2}(\mu)=\frac{1}{8} \sum_{e}^{n_{e}}(\underbrace{\epsilon^{2}(\mu) \bar{\epsilon}_{[e]}^{2}(\mu)}_{\bar{\eta}_{[e]}(\mu)}+\underbrace{\epsilon_{[e]}^{2}(\mu) \bar{\epsilon}^{2}(\mu)}_{\eta_{[e]}(\mu)})
$$

For simplicity, we will detail only with the first term $\bar{\eta}$ of Equation (47), but the computations for $\eta$ are analogous. Substituting (41) and (46) into (47) we have:

$$
\begin{aligned}
\bar{\eta}_{[e]}(\mu)=\sum_{e_{i}=1}^{n_{e}}\left(\sum_{k=1}^{m_{K}}\left[\alpha_{K}^{k}\right]_{e_{i}} \beta_{K}^{k}(\mu)+\sum_{f=1}^{m_{F}}\left[\alpha_{F}^{f}\right]_{e_{i}} \beta_{F}^{f}(\mu)-2 \sum_{s=1}^{m_{S}}\left[\alpha_{S}^{s}\right]_{e_{i}} \beta_{S}^{s}(\mu)\right) & \left(\sum_{\bar{k}=1}^{\bar{m}_{K}}\left[\bar{\alpha}_{K}^{\bar{k}}\right]_{e} \bar{\beta}_{K}^{\bar{k}}(\mu)+\sum_{\bar{f}=1}^{\bar{m}_{F}}\left[\bar{\alpha}_{F}^{\bar{f}}\right]_{e} \bar{\beta}_{F}^{\bar{f}}(\mu)-2 \sum_{\bar{s}=1}^{\bar{m}_{S}}\left[\bar{\alpha}_{S}^{\bar{s}}\right]_{e} \bar{\beta}_{S}^{\bar{s}}(\mu)\right)
\end{aligned}
$$


From $\bar{\eta}_{[e]}(\mu)$ and $\eta_{[e]}(\mu)$, we can substitute them in Equation (47). This will provide, for each set of parameters, the elemental contribution to the square of the bound of the local output error of the average solution.

We can also write the parametric version of the bound, from Equation (33), as:

$$
\epsilon_{a_{\text {local }}}(\mu)=\frac{1}{4} \sum_{e}^{n_{e}}(\underbrace{\bar{\epsilon}_{[e]}^{2}(\mu) \frac{\epsilon(\mu)}{\bar{\epsilon}(\mu)}}_{\bar{\omega}_{[e]}(\mu)}+\underbrace{\epsilon_{[e]}^{2}(\mu) \frac{\bar{\epsilon}(\mu)}{\epsilon(\mu)}}_{\omega_{[e]}(\mu)})
$$

We can substitute (41) and (46) in the first term of Equation (49), resulting in:

$$
\begin{aligned}
\bar{\omega}_{[e]}(\mu)=\left(\sum_{\bar{k}=1}^{\bar{m}_{K}}\left[\bar{\alpha}_{K}^{\bar{k}}\right]_{e} \bar{\beta}_{K}^{\bar{k}}(\mu)+\right. & \left.\sum_{\bar{f}=1}^{\bar{m}_{F}}\left[\bar{\alpha}_{F}^{\bar{f}}\right]_{e} \bar{\beta}_{F}^{\bar{f}}(\mu)-2 \sum_{\bar{s}=1}^{\bar{m}_{S}}\left[\bar{\alpha}_{S}^{\bar{s}}\right]_{e} \bar{\beta}_{S}^{\bar{s}}(\mu)\right) \\
& \left(\frac{\sqrt{\sum_{e_{i}=1}^{n_{e}}\left(\sum_{k=1}^{m_{K}}\left[\alpha_{K}^{k}\right]_{e_{i}} \beta_{K}^{k}(\mu)+\sum_{f=1}^{m_{F}}\left[\alpha_{F}^{f}\right]_{e_{i}} \beta_{F}^{f}(\mu)-2 \sum_{s=1}^{m_{S}}\left[\alpha_{S}^{s}\right]_{e_{i}} \beta_{S}^{s}(\mu)\right)}}{\sqrt{\sum_{\bar{e}_{i}=1}^{n_{e}}\left(\sum_{\bar{k}=1}^{\bar{m}_{K}}\left[\bar{\alpha}_{K}^{\bar{k}}\right]_{\bar{e}_{i}} \bar{\beta}_{K}^{\bar{k}}(\mu)+\sum_{\bar{f}=1}^{\bar{m}_{F}}\left[\bar{\alpha}_{F}^{\bar{f}}\right]_{\bar{e}_{i}} \bar{\beta}_{F}^{\bar{f}}(\mu)-2 \sum_{\bar{s}=1}^{\bar{m}_{S}}\left[\bar{\alpha}_{S}^{\bar{s}}\right]_{\bar{e}_{i}} \bar{\beta}_{S}^{\bar{s}}(\mu)\right)}}\right)
\end{aligned}
$$

The computation of the second term $\omega$ is analogous. After we compute both $\bar{\omega}_{[e]}(\mu)$ and $\omega_{[e]}(\mu)$ we can substitute them into Equation (50). This will lead to the average local output error for each set of parameters.

In terms of computing bounds, it is more interesting to use (49) than (47). This is because the squared bound is not a good bounding measure, as it does not have a physical meaning.

Neither (47) nor (49) can be used as adaptivity indicators, as each set of parameters will lead to different values of the local output error in each element. Again, a solution to this problem is to perform an integral in the parametric space of the local output error or of its squared form.

Notice that, in terms of adaptivity, it is more convenient to use the integral of (47) than the integral of (49). The first reason is because a mesh refined using the results coming from the squared error will give more importance to the regions with a higher error indicator. The second reason regards the computational implementation of these expressions. Performing the integral in the parametric domain can be computationally expensive, and the alternative to deal with this issue that we will present in the next section does not work for a squared root equation. The integral for the squared bound is:

$$
\int_{\Omega_{\mu}} \epsilon_{a_{\text {local }}}^{2}(\mu) \mathrm{d} \Omega_{\mu}=\frac{1}{8} \sum_{e=1}^{n_{e}}\left(\int_{\Omega_{\mu}}\left(\bar{\eta}_{[e]}(\mu)+\eta_{[e]}(\mu)\right) \mathrm{d} \Omega_{\mu}\right)
$$

For simplicity, we define (51) as:

$$
\Psi_{\text {local }^{2}}=\int_{\Omega_{\mu}} \epsilon_{a_{\text {local }}}^{2}(\mu) \mathrm{d} \Omega_{\mu},
$$

We will also define the total number of PGD modes $n_{m}$ in a solution as:

$$
n_{m}=\left(m_{K}+m_{F}+m_{S}\right),
$$

and $n_{M}$ the number of modes resulting for the product between a primal and virtual problem, such that:

$$
n_{M}=n_{m} n_{\bar{m}}
$$

\subsection{Implementation Details}

There are several ways to numerically compute Equations (47), (49) and (51), which involve products of PGD solutions. The optimal approach to compute these products depends on the characteristics of the problem being studied. During the course of our implementation, we noticed a few aspects that are worth mentioning, as they can largely influence the computational complexity. Each approach has its advantages and disadvantages and we will summarize them into 
two categories: when the simulations are performed in a large parametric domain and when the number of modes for the PGD approximations is large. Of course, the concept of "large" is entirely subjective, and we will try to give some examples for comparison. The results presented here are merely qualitative, as the code is not optimized for speed. The characteristics of the problem that is used are described in Section 5.

\subsubsection{Large parametric domain}

When computing $\Psi_{\text {local }^{2}}$, the size of the parametric domain affects the computational cost. If the integrals are performed numerically as in (51), it will be necessary to compute all the possible combinations of parameters in order to perform the integral, since it is the last operation performed.

This means that, for instance, a problem with three parameters and a domain composed of $n_{h}=50$ points, will have $1.25 \times 10^{5}$ combinations of parameters, which can lead to a very high computational cost. Naturally, increasing the number of integration points is desirable when seeking an accurate solution, as it will lead to a decrease in the errors coming from the numerical integration. These benefits occurs up to a certain threshold, after which other sources of error will have a greater influence in the solutions.

An alternative to (51) is to take advantage of the algebraic format of our solutions. Firstly, if we go back to Equation (48), we can manipulate it to isolate the summations in $e_{i}$ for their corresponding $[\alpha]_{e_{i}}$ and expand the product as:

$$
\begin{aligned}
& \bar{\eta}_{[e]}(\mu)= \\
& \sum_{k=1}^{m_{K}}\left(\sum_{e_{i}=1}^{n_{e}}\left[\alpha_{K}^{k}\right]_{e_{i}}\right) \beta_{K}^{k}(\mu) \sum_{\bar{k}=1}^{\bar{m}_{K}}\left[\bar{\alpha}_{K}^{\bar{k}}\right]_{e} \bar{\beta}_{K}^{\bar{k}}(\mu)+\sum_{k=1}^{m_{K}}\left(\sum_{e_{i}=1}^{n_{e}}\left[\alpha_{K}^{k}\right]_{e_{i}}\right) \beta_{K}^{k}(\mu) \sum_{\bar{f}=1}^{\bar{m}_{F}}\left[\bar{\alpha}_{F}^{\bar{f}}\right]_{e} \bar{\beta}_{F}^{\bar{f}}(\mu)-2 \sum_{k=1}^{m_{K}}\left(\sum_{e_{i}=1}^{n_{e}}\left[\alpha_{K}^{k}\right]_{e_{i}}\right) \beta_{K}^{k}(\mu) \sum_{\bar{s}=1}^{\bar{m}_{S}}\left[\bar{\alpha}_{S}^{\bar{s}^{s}}\right]_{e} \bar{\beta}_{S}^{\bar{s}}(\mu)+ \\
& \left.\sum_{f=1}^{m_{F}}\left(\sum_{e_{i}=1}^{n_{e}}\left[\alpha_{F}^{f}\right]_{e_{i}}\right) \beta_{F}^{f}(\mu) \sum_{\bar{k}=1}^{\bar{m}_{K}}\left[\bar{\alpha}_{K}^{\bar{k}_{F}}\right]_{e} \bar{\beta}_{K}^{\bar{k}_{F}}(\mu)+\sum_{f=1}^{m_{F}}\left(\sum_{e_{i}=1}^{n_{e}}\left[\alpha_{F}^{f}\right]_{e_{i}}\right) \beta_{F}^{f}(\mu) \sum_{\bar{f}=1}^{\bar{m}_{F}}\left[\bar{\alpha}_{F}^{\bar{f}}\right]_{e} \bar{\beta}_{F}^{\bar{f}}(\mu)-2 \sum_{f=1}^{\bar{m}_{S}}\left[\sum_{e_{i}=1}^{f}\right]_{e_{i}}\right) \beta_{F}^{f}(\mu) \sum_{\bar{s}=1}^{\bar{m}_{S}}\left[\bar{\alpha}_{S}^{\bar{s}}\right]_{e} \bar{\beta}_{S}^{\bar{s}}(\mu)- \\
& \left.2 \sum_{s=1}^{m_{S}}\left(\sum_{e_{i}=1}^{n_{e}}\left[\alpha_{S}^{s}\right]_{e_{i}}\right) \beta_{S}^{s}(\mu) \sum_{\bar{k}=1}^{\bar{m}_{K}}\left[\bar{\alpha}_{K}^{\bar{k}}\right]_{e} \bar{\beta}_{K}^{\bar{k}}(\mu)-2 \sum_{s=1}^{m_{S}}\left(\sum_{e_{i}=1}^{n_{e}}\left[\alpha_{S}^{s}\right]_{e_{i}}\right) \beta_{S}^{s}(\mu) \sum_{\bar{f}=1}^{\bar{m}_{F}}\left[\bar{\alpha}_{F}^{\bar{f}}\right]_{e} \bar{\beta}_{F}^{\bar{f}}(\mu)+4 \sum_{s=1}^{\bar{m}_{S}}\left[\sum_{e_{i}=1}^{s}\right]_{e_{i}}\right) \beta_{S}^{s}(\mu) \sum_{\bar{s}=1}^{\bar{m}_{S}}\left[\bar{\alpha}_{S}^{\bar{s}}\right]_{e} \bar{\beta}_{S}^{\bar{s}}(\mu)
\end{aligned}
$$

Secondly, we can rearrange the summations and move the parametric terms $\beta(\mu)$ together:

$$
\begin{aligned}
& \bar{\eta}_{[e]}(\mu)= \\
& \sum_{k=1}^{m_{K}} \sum_{\bar{k}=1}^{\bar{m}_{K}}\left(\sum_{e_{i}=1}^{n_{e}}\left[\alpha_{K}^{k}\right]_{e_{i}}\right)\left[\bar{\alpha}_{K}^{\bar{k}}\right]_{e} \beta_{K}^{k}(\mu) \bar{\beta}_{K}^{\bar{k}}(\mu)+\sum_{k=1}^{m_{K}} \sum_{\bar{f}=1}^{\bar{m}_{F}}\left(\sum_{e_{i}=1}^{n_{e}}\left[\alpha_{K}^{k}\right]_{e_{i}}\right)\left[\bar{\alpha}_{F}^{\bar{f}}\right]_{e} \beta_{K}^{k}(\mu) \bar{\beta}_{F}^{\bar{f}}(\mu)-2 \sum_{k=1}^{m_{K}} \sum_{\bar{s}=1}^{\bar{m}_{S}}\left(\sum_{e_{i}=1}^{n_{e}}\left[\alpha_{K}^{k}\right]_{e_{i}}\right)\left[\bar{\alpha}_{S}^{\bar{s}}\right]_{e} \beta_{K}^{k}(\mu) \bar{\beta}_{S}^{\bar{s}}(\mu)+ \\
& \sum_{f=1}^{m_{F}} \sum_{\bar{k}=1}^{\bar{m}_{K}}\left(\sum_{e_{i}=1}^{n_{e}}\left[\alpha_{F}^{f}\right]_{e_{i}}\right)\left[\bar{\alpha}_{K}^{\bar{k}}\right]_{e} \beta_{F}^{f}(\mu) \bar{\beta}_{K}^{\bar{k}}(\mu)+\sum_{f=1}^{m_{F}} \sum_{\bar{f}=1}^{\bar{m}_{F}}\left(\sum_{e_{i}=1}^{n_{e}}\left[\alpha_{F}^{f}\right]_{e_{i}}\right)\left[\bar{\alpha}_{F}^{\bar{f}}\right]_{e} \beta_{F}^{f}(\mu) \bar{\beta}_{F}^{\bar{f}}(\mu)-2 \sum_{f=1}^{m_{F}} \sum_{\bar{s}=1}^{\bar{m}_{S}}\left(\sum_{e_{i}=1}^{n_{e}}\left[\alpha_{F}^{f}\right]_{e_{i}}\right)\left[\bar{\alpha}_{S}^{\bar{s}}\right]_{e} \beta_{F}^{f}(\mu) \bar{\beta}_{S}^{\bar{s}}(\mu)- \\
& 2 \sum_{s=1}^{m_{S}} \sum_{\bar{k}=1}^{\bar{m}_{K}}\left(\sum_{e_{i}=1}^{n_{e}}\left[\alpha_{S}^{s}\right]_{e_{i}}\right)\left[\bar{\alpha}_{K}^{\bar{k}}\right]_{e} \beta_{S}^{s}(\mu) \bar{\beta}_{K}^{\bar{k}_{S}}(\mu)-2 \sum_{s=1}^{m_{S}} \sum_{\bar{f}=1}^{\bar{m}_{F}}\left(\sum_{e_{i}=1}^{n_{e}}\left[\alpha_{S}^{s}\right]_{e_{i}}\right)\left[\bar{\alpha}_{F}^{\bar{f}}\right]_{e} \beta_{S}^{s}(\mu) \bar{\beta}_{F}^{\bar{f}}(\mu)+4 \sum_{s=1}^{n_{e}}\left[\sum_{e_{i}=1}^{s}\left[\alpha_{S}^{s}\right]_{e_{i}}\right)\left[\bar{\alpha}_{S}^{\bar{s}}\right]_{e} \beta_{S}^{s}(\mu) \bar{\beta}_{S}^{\bar{s}}(\mu)
\end{aligned}
$$

Finally, as the parametric terms $\beta$ do not depend on the elements, we can perform the integral directly in the parametric domain, so that: 
Table 1: Speed up obtained for the computations performed using the rearranged form of $\Psi_{\text {local }}^{2}$. Results for different number of integration points $n_{h}$ and number of PGD modes.

\begin{tabular}{|c|c|c|}
\cline { 2 - 3 } \multicolumn{1}{c|}{} & \multicolumn{2}{c|}{ speed up } \\
\hline$n_{h}$ & $n_{m} \times n_{\bar{m}}=300 \times 300$ & $n_{m} \times n_{\bar{m}}=7500 \times 7500$ \\
\hline 10 & 1.611 & 0.018 \\
25 & 4.333 & 0.110 \\
50 & 14.04 & 0.432 \\
100 & 22.72 & 1.372 \\
200 & 65.97 & 4.139 \\
\hline
\end{tabular}

$$
\begin{aligned}
& \int_{\Omega_{\mu}} \bar{\eta}_{[e]}(\mu) \mathrm{d} \Omega_{\mu}= \\
& \sum_{k=1}^{m_{K}} \sum_{\bar{k}=1}^{\bar{m}_{K}}\left(\sum_{e_{i}=1}^{n_{e}}\left[\alpha_{K}^{k}\right]_{e_{i}}\right)\left[\bar{\alpha}_{K}^{\bar{k}}\right]_{e} \int_{\Omega_{\mu}}\left(\beta_{K}^{k}(\mu) \bar{\beta}_{K}^{\bar{k}}(\mu)\right) \mathrm{d} \Omega_{\mu}+\sum_{k=1}^{m_{K}} \sum_{\bar{f}=1}^{\bar{m}_{F}}\left(\sum_{e_{i}=1}^{n_{e}}\left[\alpha_{K}^{k}\right]_{e_{i}}\right)\left[\bar{\alpha}_{F}^{\bar{f}}\right]_{e} \int_{\Omega_{\mu}}\left(\beta_{K}^{k}(\mu) \bar{\beta}_{F}^{\bar{f}}(\mu)\right) \mathrm{d} \Omega_{\mu}- \\
& 2 \sum_{k=1}^{m_{K}} \sum_{\bar{s}=1}^{\bar{m}_{S}}\left(\sum_{e_{i}=1}^{n_{e}}\left[\alpha_{K}^{k}\right]_{e_{i}}\right)\left[\bar{\alpha}_{S}^{\bar{s}}\right]_{e} \int_{\Omega_{\mu}}\left(\beta_{K}^{k}(\mu) \bar{\beta}_{S}^{\bar{s}}(\mu)\right) \mathrm{d} \Omega_{\mu}+\sum_{f=1}^{m_{F}} \sum_{\bar{k}=1}^{\bar{m}_{K}}\left(\sum_{e_{i}=1}^{n_{e}}\left[\alpha_{F}^{f}\right]_{e_{i}}\right)\left[\bar{\alpha}_{K}^{\bar{k}}\right]_{e} \int_{\Omega_{\mu}}\left(\beta_{F}^{f}(\mu) \bar{\beta}_{K}^{\bar{k}}(\mu)\right) \mathrm{d} \Omega_{\mu}+ \\
& \sum_{f=1}^{m_{F}} \sum_{\bar{f}=1}^{\bar{m}_{F}}\left(\sum_{e_{i}=1}^{n_{e}}\left[\alpha_{F}^{f}\right]_{e_{i}}\right)\left[\bar{\alpha}_{F}^{\bar{f}}\right]_{e} \int_{\Omega_{\mu}}\left(\beta_{F}^{f}(\mu) \bar{\beta}_{F}^{\bar{f}}(\mu)\right) \mathrm{d} \Omega_{\mu}-2 \sum_{f=1}^{m_{F}} \sum_{\bar{s}=1}^{\bar{m}_{S}}\left(\sum_{e_{i}=1}^{n_{e}}\left[\alpha_{F}^{f}\right]_{e_{i}}\right)\left[\bar{\alpha}_{S}^{\bar{s}}\right]_{e} \int_{\Omega_{\mu}}\left(\beta_{F}^{f}(\mu) \bar{\beta}_{S}^{\bar{s}}(\mu)\right) \mathrm{d} \Omega_{\mu}- \\
& 2 \sum_{s=1}^{m_{S}} \sum_{\bar{k}=1}^{\bar{m}_{K}}\left(\sum_{e_{i}=1}^{n_{e}}\left[\alpha_{S}^{s}\right]_{e_{i}}\right)\left[\bar{\alpha}_{K}^{\bar{k}}\right]_{e} \int_{\Omega_{\mu}}\left(\beta_{S}^{s}(\mu) \bar{\beta}_{K}^{\bar{k}}(\mu)\right) \mathrm{d} \Omega_{\mu}-2 \sum_{s=1}^{m_{S}} \sum_{\bar{m}=1}^{\bar{m}_{F}}\left(\sum_{e_{i}=1}^{n_{e}}\left[\alpha_{S}^{s}\right]_{e_{i}}\right)\left[\bar{\alpha}_{F}^{\bar{f}}\right]_{e} \int_{\Omega_{\mu}}\left(\beta_{S}^{s}(\mu) \bar{\beta}_{F}^{\bar{f}}(\mu)\right) \mathrm{d} \Omega_{\mu}+ \\
& 4 \sum_{s=1}^{m_{S}} \sum_{\bar{s}=1}^{\bar{m}_{S}}\left(\sum_{e_{i}=1}^{n_{e}}\left[\alpha_{S}^{s}\right]_{e_{i}}\right)\left[\bar{\alpha}_{S}^{\bar{s}}\right]_{e} \int_{\Omega_{\mu}}\left(\beta_{S}^{s}(\mu) \bar{\beta}_{S}^{\bar{s}}(\mu)\right) \mathrm{d} \Omega_{\mu} \quad(57)
\end{aligned}
$$

Using Equation (57) simplifies the integrals, as in this format they can be computed as one dimensional products of integrals. The drawback of this approach is that it is now required to perform products between modes of the real and virtual solutions, which can lead to a very high total number of PGD modes $n_{M}$. This product is not mandatory if we work with Equation (51). Another drawback is that we cannot extend this approach to the integral of (49), as the integral of the square root is not the square root of the integral.

Table 1 presents the speed up obtained by using Equation (57) instead of (48), with different number of points $n_{h}$ to describe the parametric domain and different number of PGD modes, with $n_{e}$ equal to 20 elements. It is observed that the speed up increases quickly as we increase the number of points. One important aspect is that the performance of the simulations using the integrals as in (57) are largely influenced by the number of modes for the PGD approximations. This method is ideal when the ratio between the number of points and the expected number of modes in the simulation is large enough. The number of elements in the mesh does not seem to have a relevant influence in the variation of the speed up.

\subsubsection{Large number of modes}

The computation of $\epsilon_{a_{\text {local }}}^{2}$ requires a product between two quantities that are approximated using a PGD, resulting in $n_{m}$ or $n_{\bar{m}}$ modes. When the form of the integral (57) is used, these quantities are further multiplied. This means that the total number of modes $n_{M}$ to compute the integral of the local output error can increase rapidly. For instance, a problem with a single parameter, in which the displacements $\hat{\mathbf{u}}$ and stresses $\hat{\mathbf{s}}$ for the real and virtual problem are 
described using 20 PGD modes will result in:

$$
\begin{aligned}
n_{M} & =n_{m} \times n_{\bar{m}} \\
& =\left(m_{K}+m_{F}+m_{S}\right)\left(\bar{m}_{K}+\bar{m}_{F}+\bar{m}_{S}\right) \\
& =\left(n_{k}^{2} n_{\mathcal{C}}+n_{s}^{2} n_{C}+n_{k} n_{s}\right)\left(n_{\bar{k}}^{2} n_{\mathcal{C}}+n_{\bar{s}}^{2} n_{C}+n_{\bar{k}} n_{\bar{s}}\right) \\
& =\left(20^{2} \times 1+20^{2} \times 1+20 \times 20\right)\left(20^{2} \times 1+20^{2} \times 1+20 \times 20\right) \\
& =1.44 \times 10^{6}
\end{aligned}
$$

The PGD procedure does not guarantee orthogonality between the terms of the result. Therefore, it is usually possible to reduce the amount of terms, without any significant loss of information. This procedure is usually called compression. Minimizing the number of terms is always desirable as most operations with separated objects scale linearly in computational cost with the number of terms.

One way to perform compression is by doing a least-square projection of the solution into the same approximation space using the same PGD procedure [27, 20, 32]. Although this does not impose orthogonality between the terms, in practice it usually reduces the number of terms of a separated function.

When and where to apply the compression procedure is a tradeoff that involves computational time and accuracy. Compressing a scalar quantity is usually easier than a vectorial one. Compression reduces the number of terms and therefore operations take shorter, although, the compression procedure also requires some non negligible time, and therefore some tradeoff must be found. Taking this into consideration we applied the compression procedure on the output from (48) which is a scalar and therefore will require less information to be described. Table 2 presents the new number of modes $n_{m^{c}}$ and $n_{\bar{m}^{c}}$ obtained for the real and virtual solutions after using the compression for all elements at once.

Table 2: Number of modes obtained for the computations performed using the pgdCompression routine. Results for different number of modes $n_{m}$ and $n_{\bar{m}}$, and different number of mesh elements $n_{e}$.

\begin{tabular}{|c|c|}
\hline$n_{m} \times n_{\bar{m}}$ & $n_{m^{c}} \times n_{\bar{m}^{c}}$ \\
\hline $300 \times 300$ & $20 \times 14$ \\
$1875 \times 1875$ & $19 \times 14$ \\
$7500 \times 7500$ & $21 \times 14$ \\
$30000 \times 30000$ & $21 \times 14$ \\
\hline
\end{tabular}

The number of modes obtained using the compression is smaller than the number of PGD modes in the original solution. It is also noticeable that increasing the number of modes in the original solution has little influence in the resulting number of compressed modes, since the additional modes do not significantly affect the final compressed solution. The relative difference of the results with and without compression is also dependent on the tolerance of the compression process, but is well within an acceptable range, with the default tolerance of $1 \times 10^{-3}$ leading to results with less than $0.0002 \%$ difference. This relative difference depends on the complexity of the problem being studied, but by decreasing the compression tolerance we can decrease the relative difference values, with the drawback of requiring more modes in the compressed solutions.

A drawback of using the compression in the context of the dual error analysis is that the solutions bounding properties are lost, since the constraints that enforce continuity are disregarded during the compression. This can be a problem when looking for specific solutions, however, a sufficient small tolerance for the compression process will still result in accurate results.

\section{Results}

The examples presented in this section intend to demonstrate how the proposed procedures can efficiently provide guaranteed bounds of local outputs. We also assess the influence of using an error indicator associated with a local quantity to drive an adaptive refinement process. 
We use the simple academic problem described in Figure 1. It corresponds to the symmetry simplification of a square plate subjected to an unit horizontal traction. The plate is composed of two sections $S$, each made of materials with different Young's modulus $E$ defined in a range [0.1,2.1]. The Poisson ratio $\nu$ for both sections is fixed at 0.15 .

Simulations with a more complex geometry were performed, but their results are not significantly different from those obtained for the simpler problem. Hence, we decided to present only the results from the square plate problem because it is easier to focus on the aspects that affect the goal-oriented refinement and the PGD approximations, such as the change of signs in the results, depending on the set of parameters chosen for the Young's moduli. Several applications using a more complex geometry, in the context of adaptivity driven by the global error, can be found in a previous paper [29].

The PGD solutions obtained are always converged in terms of number of modes, in order to consider only the errors sources coming from the mesh.

Although the stopping criteria used by the algebraicPGDtools code are a reliable way to determine when the PGD solution has converged, since we are independently computing pairs of solution, it may lead to more modes than necessary for our application $[27,14]$. Hence, we use the information we have of the energy of the error as our PGD stopping criterion, assessing the convergence of the PGD approximations, considering the pair of solutions. The criterion is obtained by computing, for each additional mode of the PGD, the value of Equation (43) and divide it by the energy of the system. We then compare the relative difference with the previous solutions and stop the process when the difference reaches a certain threshold tolerance $\tau$. Note that this means that both solutions of the global problem will have the same number of PGD modes. Furthermore, this criterion is applicable for either the real or the virtual problem.

This verification, which is performed for every additional PGD mode, becomes more costly as the number of modes increases, remaining a small fraction of the total running time. It is worth mentioning that computing the equilibrated solutions is usually more costly than computing the compatible approximations, by a factor close to two, for both the pre-processing and the solution of the PGD. This is highly dependent on the size of the finite element mesh, increasing for larger meshes. Naturally, these are just rough estimates, as the codes used to obtain these solutions are not aimed for efficiency.

To stop the adaptivity refinement process we consider either if the ratio between the error for the current mesh and the energy of the problem is bellow a certain threshold or if a given number of elements in the mesh is exceeded. A more detailed explanation on the stopping criteria used can be found in our previous work on global adaptivity for PGD solutions [29].

The parametric domain, unless otherwise stated, has $n_{h}=50$ points. The tolerance for the PGD model, the fixed point iteration and the adaptivity process are all set to $10^{-4}$. As we will demonstrate at the end of this section, this value guarantees that the error from the PGD approximations is negligible. The maximum number of fixed points iterations is 15 , which may be low for more complex problems but, as we are working with an smooth problem, gives good results without slowing the computations. If during the mesh refinement process the tolerance is not reached, the adaptivity process stops when the mesh exceed $n_{e}=6000$ elements. This number of elements is chosen due to computational constraints. The degree of the polynomial approximations in space are always linear for the equilibrated solutions and quadratic for the compatible ones, so that the stress fields of both solutions are described by linear functions.

We consider two different QoIs:

- The self compliant problem associated with the horizontal load, where the QoI is the integral of the horizontal displacement at the right side of the plate. For this QoI the real and virtual load are the same, being represented by the full arrows in Figure 1;

- The integral of the vertical displacement on a line at top boundary of the plate, due to the horizontal load. Here, the real load corresponds to the full arrows, and the virtual loads to the hollow arrows in Figure 1.

Figure 1 also presents the displacement fields obtained for the global problem when Young's moduli at the opposite edges of the parametric domain are considered, for a reference case with polynomial degrees of approximation of the space equal to 6 and 7 for equilibrated and compatible solution, respectively, $n_{e}=320$ elements, $n_{h}=50$ and a number of modes equal to 50 for the real and virtual solutions. The number of modes used for this specific simulation is arbitrarily high, so that the errors shown are based only on the mesh. All the other simulations have a variable number of modes, which are controlled by the selected tolerance. 


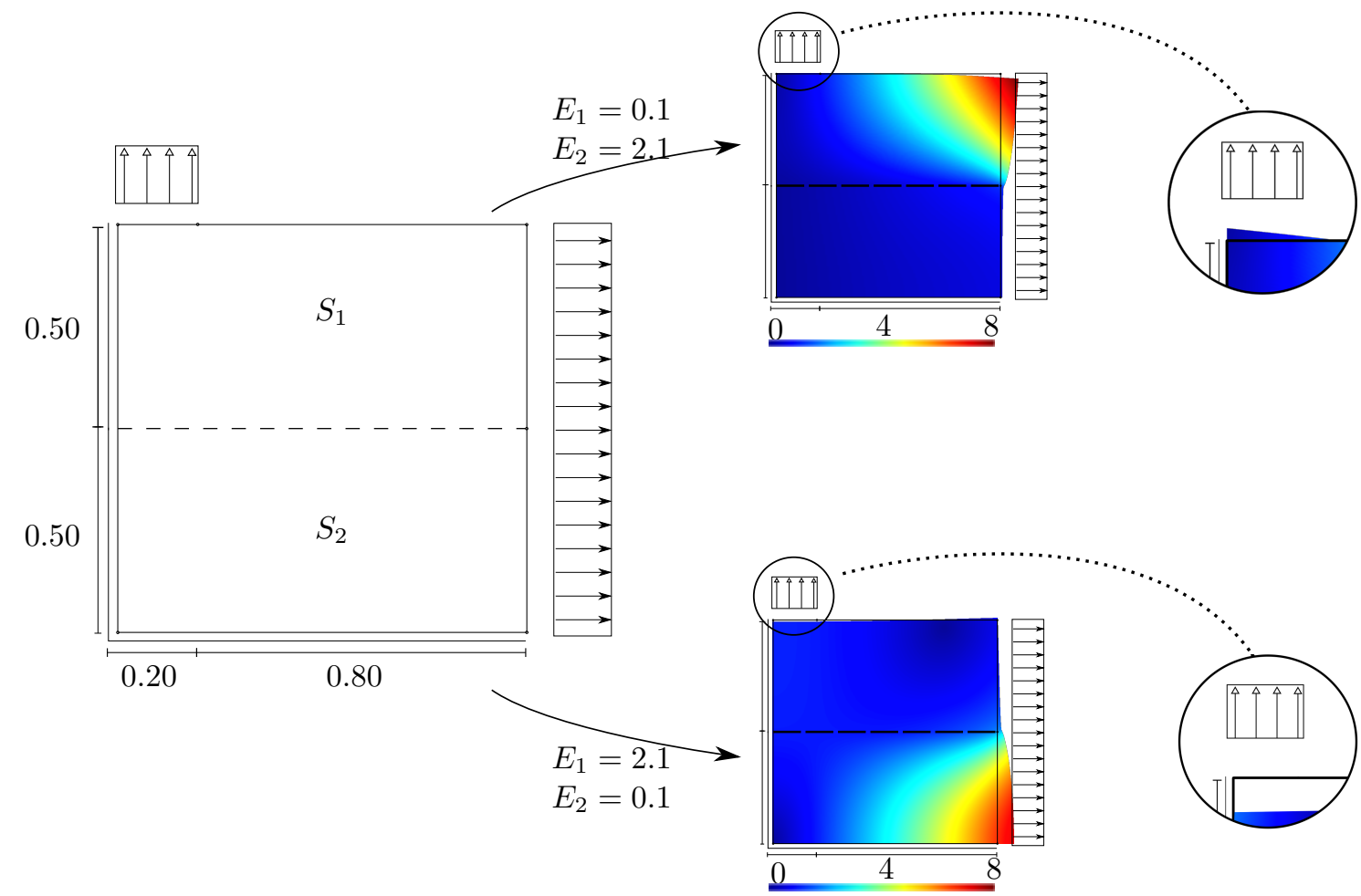

Figure 1: Symmetric simplification of a square plate, with the representations of the real (solid arrows) and virtual (hollow arrows) loads for the second QoI. The displacement fields for the case with the Young's moduli at the opposite edges of the parametric domain are presented, with details for the region where the displacement changes signs. The displacements at the detail plots are exaggerated for a better visualisation.

As shown in the detail of the figure, this problem is particularly interesting because the output changes sign and, consequently, it is zero for certain values of the parameters. This is important because, when the outputs are obtained from a poor solutions, the information obtained may be grossly misleading. The only way to be certain of the quality of the output is by looking at its bounds. Also note that, because the output is zero for certain combinations of the parameters, it is not reasonable to work with the relative error.

\subsection{The Self-Compliant Problem}

We begin with a self-compliant problem, comparing the results obtained by applying either a global or a QoI driven adaptivity technique. The apparent equivalence between both approaches is discussed, and the results are used as a validation of the codes used.

When using the same load for the real and for the virtual problem the energies are the same, so that the local output bounds are proportional to the global energy. This can be demonstrated by using the same value for the bound of the energy of the error in Equations (47) or (49) for the real and virtual problems.

Figure 2 presents the values obtained for the integrals of the global (left) and local output (right) errors using refinements driven by the global or local output error indicators. As expected, the values obtained are practically identical. Note that the slope for $\Psi_{\text {global }}{ }^{2}$ is almost twice that for $\Psi_{\text {local }}{ }^{2}$, which is expected, because the local output indicator is based on a product of two energies.

The difference in the results for either integrals come from the fact that the meshes used by the refinements driven by the local output and global indicators are different. This is because, although it may seem that the integrals $\Psi_{\text {global }}{ }^{2}$ 

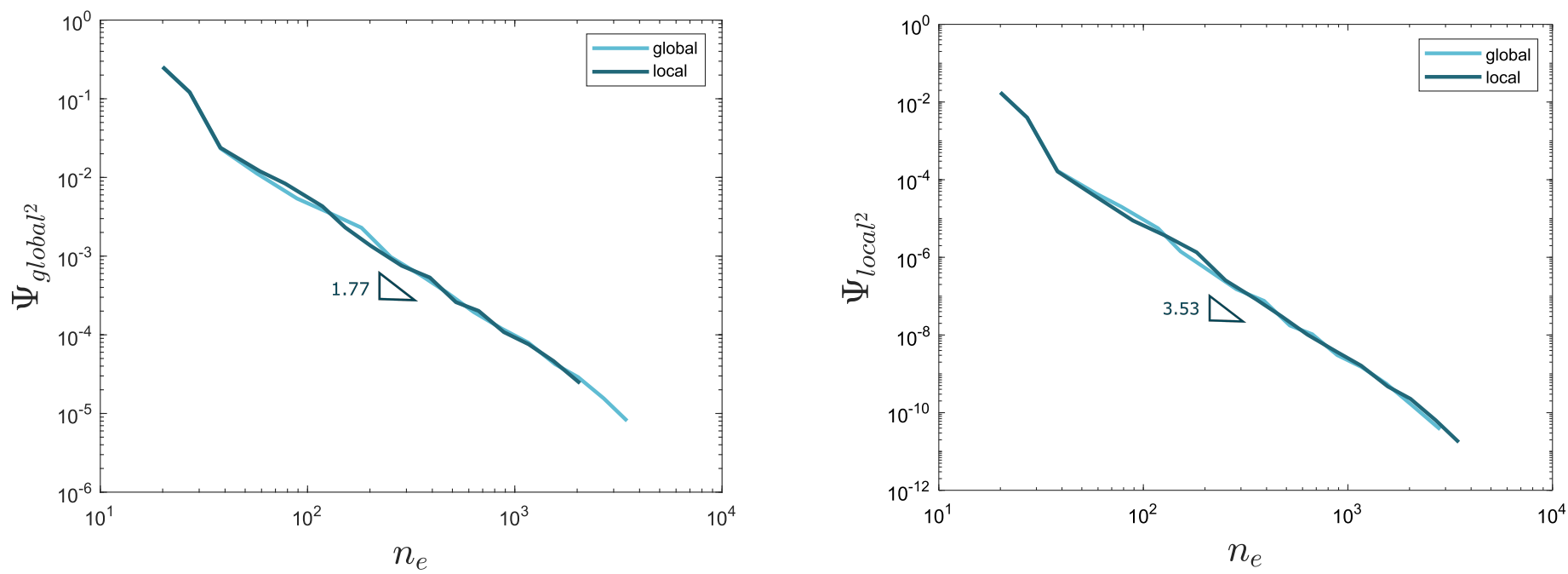

Figure 2: Self-compliant problem. The integrals of the global (left) and local output (right) errors as a function of the number of elements in the mesh. We present the cases of errors for the sequence of meshes obtained with an adaptive refinement based on the local output error indicator and a refinement driven by the global error indicator.

and $\Psi_{\text {local }}{ }^{2}$ have only a difference in scale, in reality one cannot immediately be obtained from the other, as the integral of the square of a function is not the square of its integral.

\subsection{The Displacement at the Top as the QoI}

For this problem we present in Figure 3 the meshes obtained by using a uniform mesh refinement and by driving the adaptivity process with the integrals of the global or local output error indicators. We observe that:

- The uniform mesh refinement process has no information regarding the quality of the solution and, consequently, the final mesh obtained has the highest error and no particular distribution of the elements;

- The mesh obtained by using the global error indicator has information of the error of the solution of the real problem and captures the transition of mechanical properties, with an error that is much lower than that obtained with uniform refinement;

- The mesh resulting from using the local output error indicator is capable of capturing both the mechanical singularity and the effect of the virtual load in the solution of the problem, leading to the lowest values of the local output error.

Notice that the number of elements $n_{e}$ for the final meshes is not the same and hence the error values obtained cannot be compared directly, only in a qualitative manner.

We summarize in Figure 4 the values obtained for the integrals of the global and local output errors using the different types of refinement. As expected, using either the global or the local output error indicators is always better than uniformly refining the mesh, since having information about the error results in more elements being used where they lead to lower error values. Also, using the local output error indicator leads to better results for the local output error while using the global error indicator yields better results in the global error values.

This effect is well defined in a sufficiently smooth problem, such as the one that we are studying. However, this may not be the case for other problems, particularly when other error sources influence the solutions, for example geometrical singularities. It was observed that when working with local outputs for the example in [29], the meshes obtained based on the global or local output adaptivity indicators are very similar.

We present in Figure 5 the local output errors obtained from the different refinements when considering the different combinations for the values of the Young's modulus E. The meshes used are the most refined ones presented in Figure 


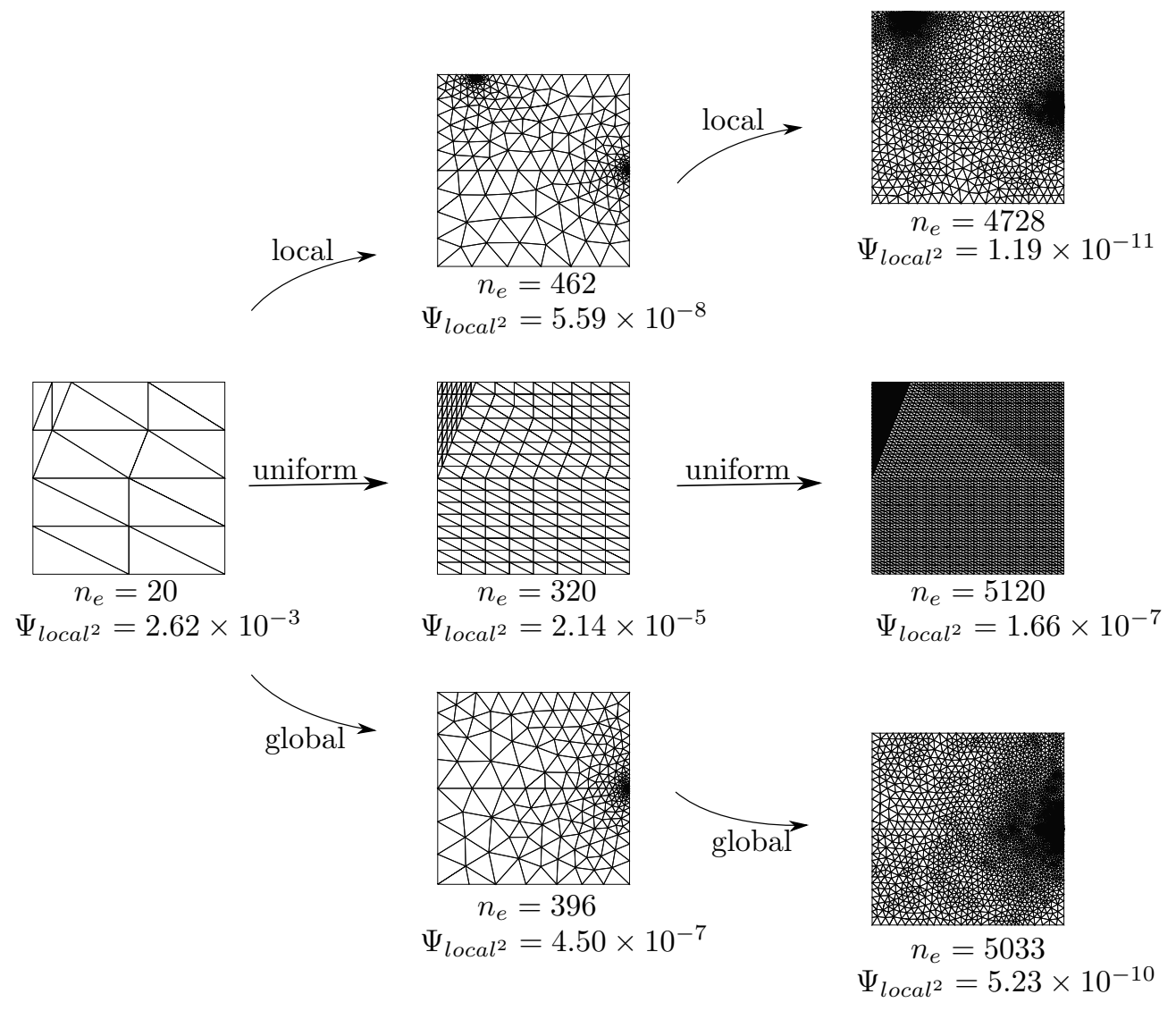

Figure 3: The meshes obtained during the adaptive refinement process using the displacement at the top as the QoI. We consider the cases of a refinement driven by the local output error indicator (top), a uniform mesh refinement (middle) and an adaptive refinement process driven by the global error indicator (bottom). 

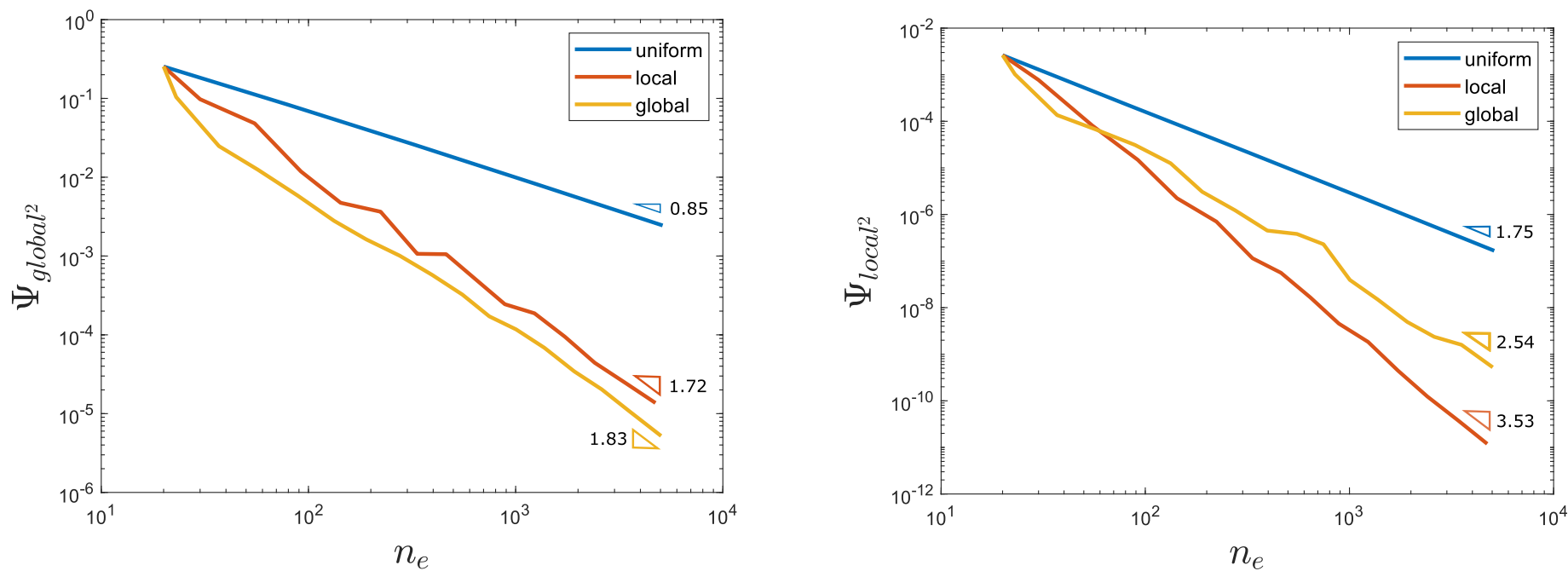

Figure 4: The integrals of the global (left) and local output (right) errors as a function of the number of elements in the mesh, using the displacement at the top as the QoI. We present the cases of errors for the sequence of meshes obtained with a uniform refinement, an adaptive refinement based on the local output error indicator and a refinement driven by the global error indicator.

3. Using the uniform refinement yields the worse results, even though its mesh has the highest number of elements, while driving the refinement with the local output error indicator provides the best results, even with fewer elements. The edges of the parametric domain have the worse approximations, particularly when one of the sections has a lower Young's modulus, because that results in higher relative displacements between section.

The lowest values for the error are in the region where the materials of the two sections are the same. In this region, the exact stresses and strains are uniform and, hence, can be represented by the finite element solution so that the error should be zero. This is not the case because the PGD introduces truncation errors, still the error in this regions is lower.

Figure 6 presents the output and its bounds for an initial mesh (top), an uniformly refined mesh (left) and a mesh obtained by using the local output error indicator to drive the adaptivity process (right), always using the same range in similar plots. The figure also presents the output for the refined meshes for the case when the Young's modulus of the second section is $E_{2}=1$, detailing the region when both moduli are equal.

Again, the edges of the parametric domain have a much higher influence in the output obtained, with the bounds in the initial mesh being much higher when one the sections has a soft material. Using the goal-oriented refinement lead to a larger improvement in the quality of the bounds than the uniform refinement. This behaviour is better visualized in the plot of the cross section, where we see in the detail that, for the case where both the materials are equal, the uniform refinement has larger errors than the refinement based on the local output error adaptivity indicator. The number of elements $n_{e}$ in both meshes is different $\left(n_{e}=80\right.$ for the uniform and $n_{e}=92$ for the adapted), but the qualitative analysis still holds.

Notice that the sign of the output for the lowest values of $E_{1}$ is still uncertain in the bounds provided by both meshes, indicating that further refinement is necessary.

Finally, we combine in Figure 7 the local output obtained from fine solutions, with a high number of elements and points in the parametric domain $\left(n_{e}=1280, n_{h}=100\right)$, which can be considered a reference solution, with the bounds of the local output obtained from a coarse solution $\left(n_{e}=20, n_{h}=5\right)$.

It is observed that at the points of parametric discretization, some of which are detailed in the figure, the bounds are always effective. However, at points of the parametric domain that were not discretized, this is not always the case. 


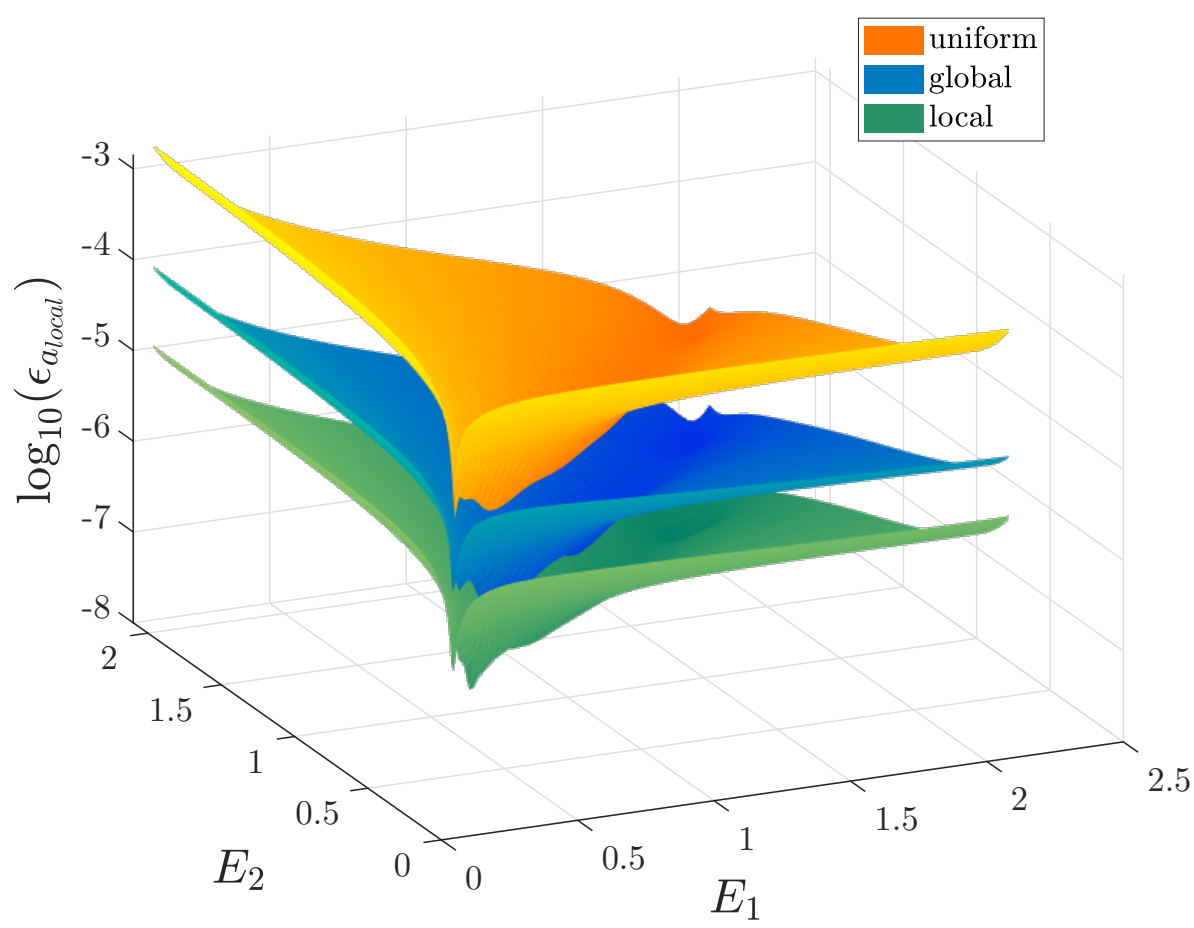

Figure 5: Error bounds of the displacement at the top for all the combinations of parameters values. We present the cases where the meshes obtained by a uniform refinement, a refinement based on the global error indicator and a refinement driven by the local output error indicator. The meshes used are the most refined ones presented in Figure 3 


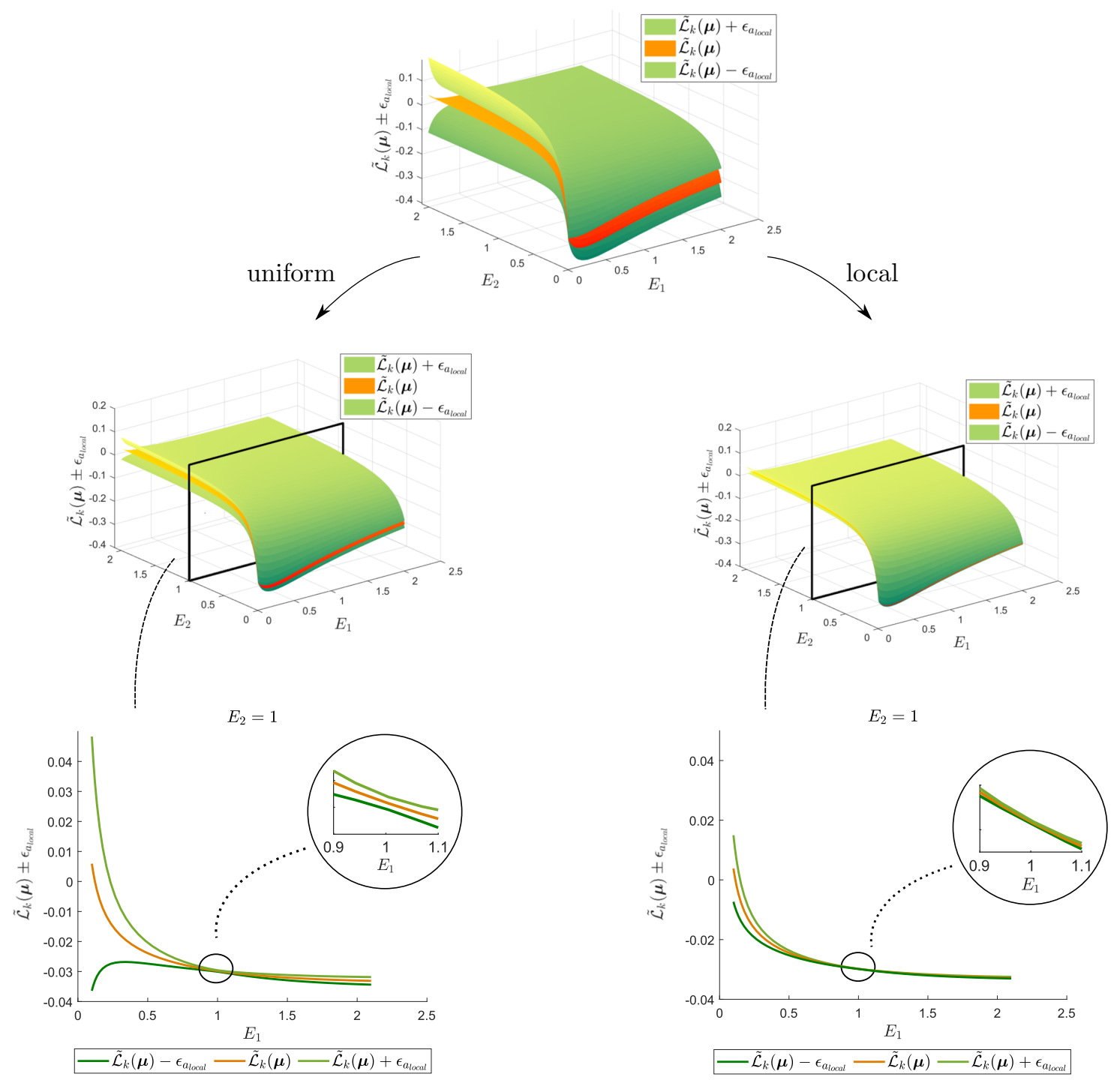

Figure 6: Displacements outputs and their respective bounds for all the possible parameters values. We present the case for the outputs and bounds obtained with an initial mesh (top) and the cases with a uniformly refinement mesh (left) and a mesh refined using the local output error indicator (right). The details in the uniformly and locally refined meshes present the values of the output and bounds when the Young's modulus $E_{2}=1$ and the regions where the two moduli are equal. 


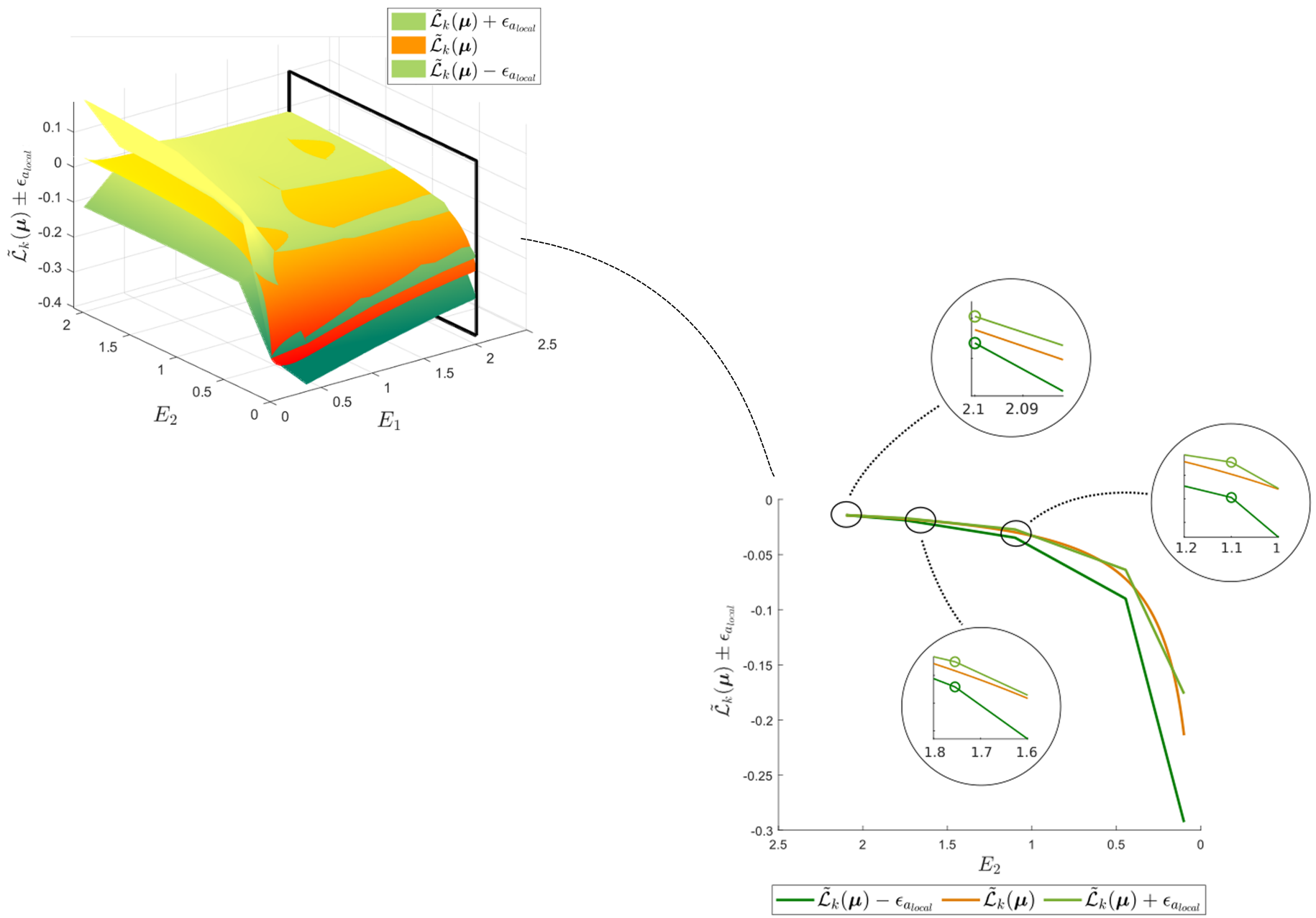

Figure 7: A reference local displacement output combined with the bounds of the local output obtained from a coarse mesh. The details present the case where the Young's modulus $E_{1}=2.1$ and the points where the parametric domain is discretized. 

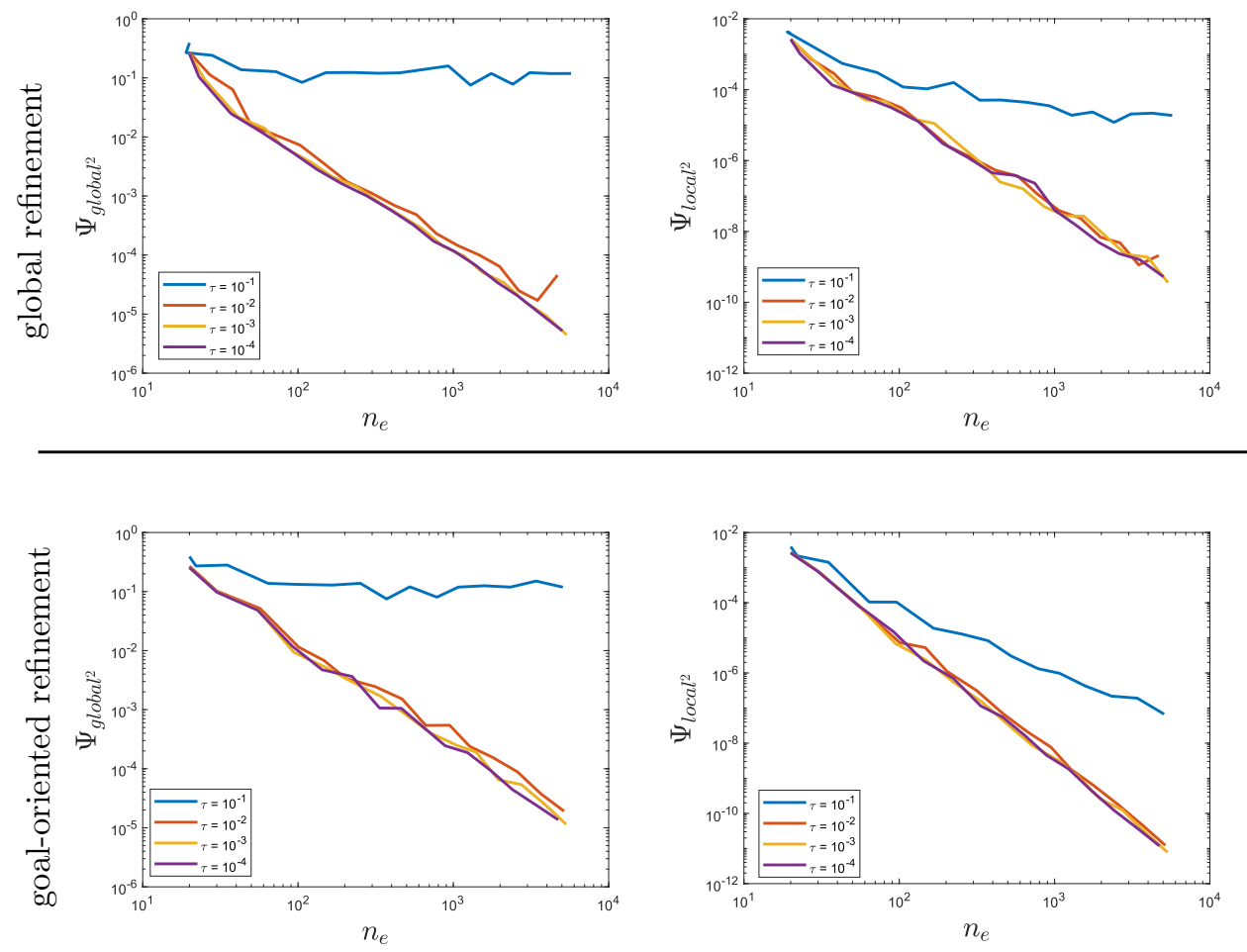

Figure 8: The integrals of the global (left) and local output (right) error bounds as a function of the number of elements in the mesh, using the displacement at the top as the QoI. We present the cases where the PGD approximations have different $\tau$ values, for refinements driven by the global error indicator (top) and by the local output error indicator (bottom).

\subsection{The effect of PGD convergence on mesh refinement}

In this section we assess how the convergence of the PGD, controlled by tolerance $\tau$, affects the mesh refinement process. We run two different examples, the refinement driven by the global error indicator and the refinement driven by the local output error indicators, each one with tolerances for the PGD approximations $\tau$ that vary from $10^{-1}$ until $10^{-4}$.

We present the results in terms of the integrals $\Psi$ in Figure 8, with the values seen for the tolerance of $10^{-4}$ are the same seen in Figure 4. First and foremost, we note that even for the less strict tolerance values, which do not seem to converge, we always obtain upper bounds for the integrals of the errors (global or local output). The downside of using less strict tolerances is having bounds that are excessively large, although this does not mean that the meshes obtained do not take advantage of the knowledge from the error in each element.

The final meshes obtained from each refinement are shown in Figure 9. From this figure we can see that, even for a tolerance value of $10^{-1}$, the key features of the problem, such as the material singularities, are captured. Naturally, the accuracy is much lower than the one obtained for the meshes that use a tolerance of $10^{-4}$. This is reflected in the density of elements in the less refined zones.

The gray dashed lines in Figure 10 show, for the goal-oriented refinement process, what happens when, from a mesh refined with a tolerance of $10^{-1}$, we compute the PGD solution and $\Psi$ with a tolerance of $10^{-4}$. This supports the idea that the meshes obtained from coarse PGD solutions are able to capture the most important information from the problem. If we continue the mesh refinement, we will eventually match the results obtained by using a tolerance of $10^{-4}$ from the beginning. This is shown, for two specific solutions, by the green and yellow dashed lines in that 

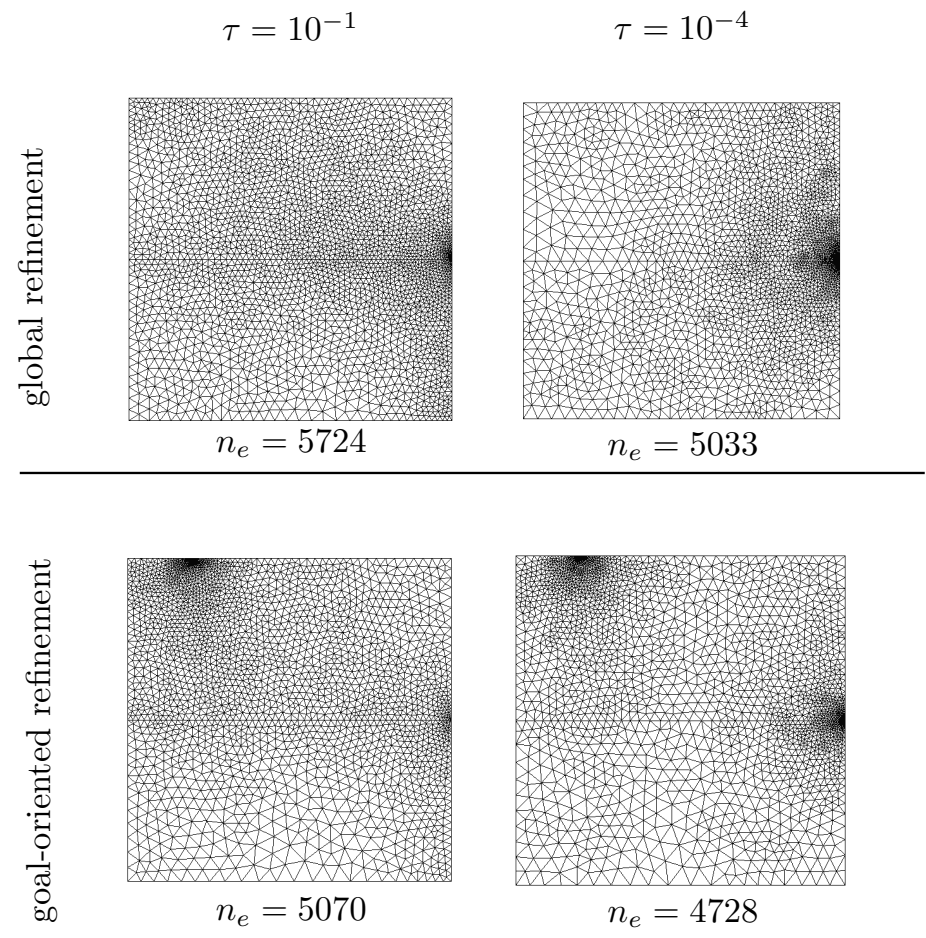

Figure 9: The final meshes obtained during the adaptive refinement process using the displacement at the top as the QoI, considering different $\tau$ tolerances for the PGD approximations. We consider the cases of a refinement driven by the global error indicator (top) and by the local output error indicator (bottom). 

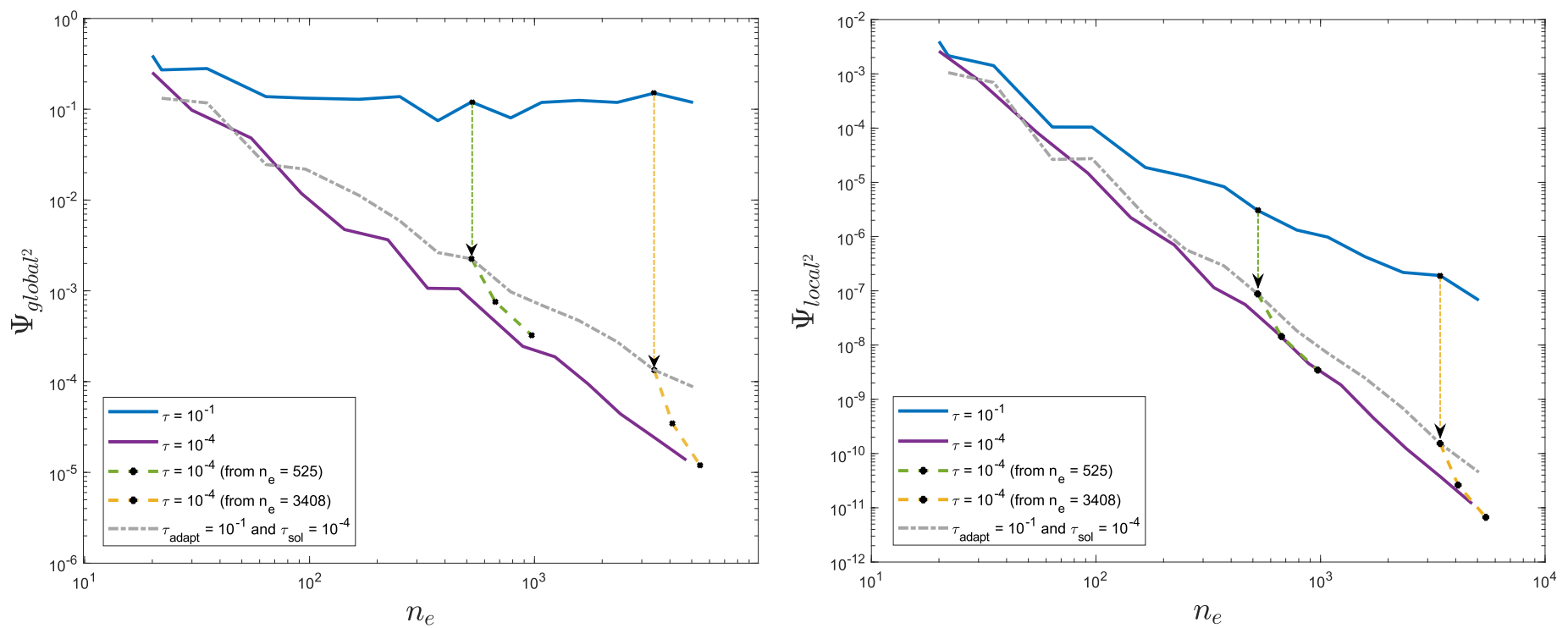

Figure 10: Details for the integrals of the global (left) and local output (right) errors as a function of the number of elements in the mesh, for the goal-oriented refinement process using the displacement at the top as the QoI. We present the cases where the PGD approximations have different $\tau$ values, and the case where the bounds are obtained with better tolerances than the ones used in the adaptivity process. Details are given for the values obtained by changing the tolerance after $n_{e}=525$ and $n_{e}=3408$.

figure, where we differentiate between the PGD tolerance used during the mesh adaptivity process, and the one that is used to compute the outputs, we use respectively $\tau_{\text {adapt }}$ and $\tau_{\text {sol }}$. Note that, before Figure 10, we always considered $\tau=\tau_{\text {adapt }}=\tau_{\text {sol }}$.

In Figure 11 we present the error bounds of the displacement outputs obtained with the finest meshes from Figure 10 (as in Figure 5) in this case using different values for $\tau_{\text {adapt }}$ and $\tau_{\text {sol }}$. This result confirms the global behavior in Figure 10 , however it should be noted that for some values of the parameters the non-optimal mesh leads to smaller errors, as can be observed by the blue regions below the green surface. These are located in the regions where $E_{1} \approx E_{2}$, where the problem is nearly homogeneous and the error of the finite element solution is zero. The homogeneous behavior is not captured by the PGD approximations, resulting in marginally different solutions for different meshes.

The behavior demonstrated in Figure 10 and 11 opens the question of whether it is possible to use a larger tolerance at the beginning of the computations, which is increased after a certain number of steps. The figures seem to indicate that this is a valid option. The unsolved question is how to define when to modify the tolerance. If we truncate too soon, we may have the error coming from the PGD approximations influencing the refinement. Also, if the tolerance is too large, we may completely lose the convergence of the refinement process.

Following this reasoning it would be desirable to have a procedure that controls the total computational cost of the adaptive refinement process, which will strongly depend on the implementation.

We note, however, that the consideration in the initial steps of more optimistic, although less strict, bounds may be a way to move forward, since the availability of a guaranteed interval of the output is critical only for the final mesh.

\section{Conclusion}

In this paper dual error analysis is applied to PGD solutions to compute local outputs and bounds of their values. We use the elemental contributions to the error of the local output to compute a novel adaptivity indicator, which is capable of producing meshes with much lower errors of the local output than those obtained with a uniform refinement. The proposed method is particularly interesting when a high quality solution is required, which is the case for most 


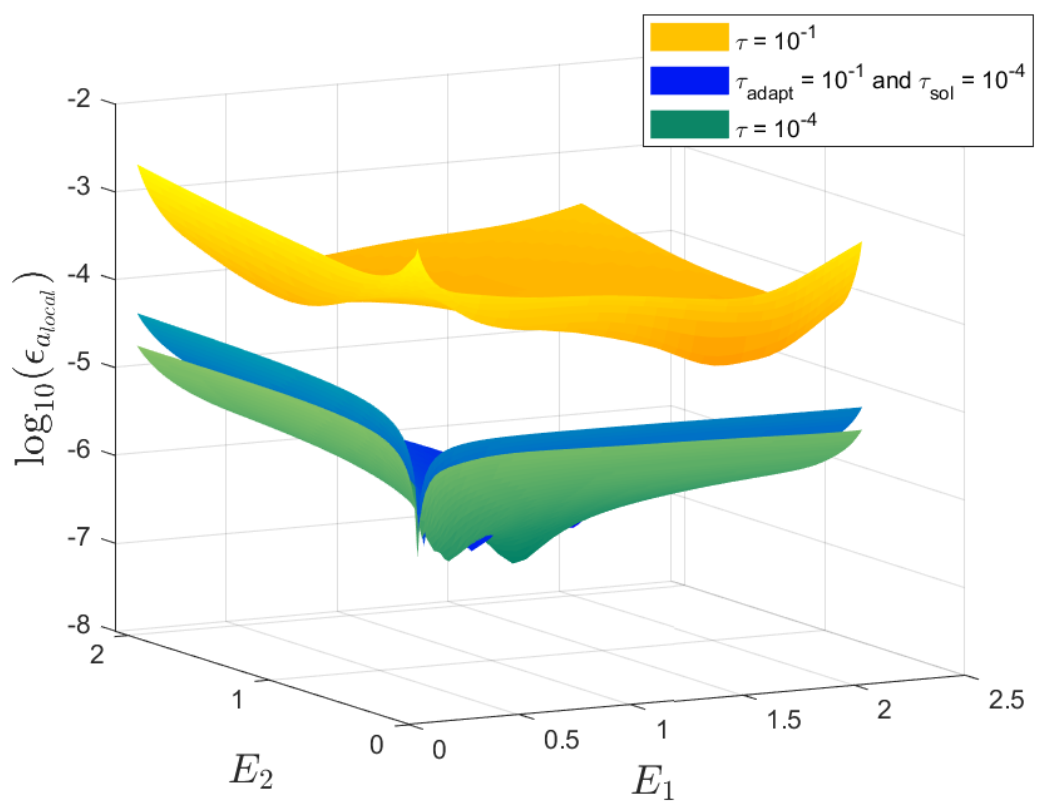

Figure 11: The values for the error bounds of the displacement at the top for all the combinations of parameters values. We present the cases where the PGD approximations have different $\tau$ values, and the case where the output solutions are obtained with a better tolerance then the one used in the adaptivity process. The meshes used are the most refined ones presented in Figure 9 for the goal-oriented refinement. 
design and engineering problems.

We study a two dimensional linear elastic problem, demonstrating how to obtain compatible and equilibrated solutions for this problem. These complementary solutions are combined to compute global error bounds, local outputs and bounds of their error. We explain how the outputs for displacements can be obtained, with bounds calculated using the average of the complementary solutions.

The problem is then cast into a parametric framework and we demonstrate how to use the algebraicPGDtools solver to obtain separable PGD parametric solutions. These solutions are used to write the parametric form of the error bounds and local output, while considering how to take advantage of the separated form of the tensors provided by the algebraicPGDtools to speed up the computations. The advantages and disadvantages of compressing the tensors describing the PGD solutions are also discussed.

We propose an adaptivity indicator based on the parametric expressions used to compute the bounds of the error of the local output, which is capable of identifying the regions in the mesh that need to be refined in order to produce lower local output errors.

We apply the concepts described to an academic problem which has the characteristic of resulting in solutions where, depending on the parameters, the local output can have opposite signs. This type of behavior highlights the importance of having reliable bounds as the wrong definition of a sign can lead to misleading conclusions.

We focus on assessing the quality of the outputs obtained from meshes derived by adaptive refinements based on either the global or the local output error indicators. The local output error indicator proved to be efficient in capturing the nature of the local quantity being studied, leading to meshes with the lowest values of the error of the local output, when compared with a uniform refinement and with the use of a global error indicator.

We use the meshes obtained with different refinement criteria to present the output and bounds of its error for all the combinations of parameters, detailing critical zones for the simulation such as the edges of the parametric domain. We also assess the region where the errors should be the lowest, which is when the parameters have equal values. Finally, we demonstrate how the bounds for a reference local output are guaranteed as long as they coincide with the points of parametric discretization.

The proposed approach can be applied to other problems. We envisage, namely, to consider:

- How to implement a variable tolerance during the refinement process. The critical point is how to achieve the target error, given the excessively large bounds that are obtained when the tolerance has a large value.

- Three-dimensional elastic problems. This requires efficiently computing equilibrated solutions in 3D, which is an extension of the formulation proposed in [18].

- Mesh adaptivity for multiple loads and multiple outputs. This can be achieved by either considering a generalization of the combined error proposed in [6] or simply the minimal mesh element size approach proposed by Zienkiewicz [31].

\section{Acknowledgements}

Jonatha Reis was supported by the European Education, Audiovisual and Culture Executive Agency (EACEA) under the Erasmus Mundus Joint Doctorate "Simulation in Engineering and Entrepreneurship Development (SEED)", FPA 2013-0043.

Pedro Díez and Sergio Zlotink are grateful for the financial support provided by the Spanish Ministry of Economy and Competitiveness (Grant agreement No. DPI2017-85139-C2-2-R), the Generalitat de Catalunya (Grant agreement No. 2017-SGR-1278), and the project H2020-RISE MATH-ROCKS GA n⿳⺈ 777778.

\section{Conflict of Interest}

The authors declare that they have no conflicts of interest. 


\section{References}

[1] I. Alfaro, D. González, S. Zlotnik, P. Díez, E. Cueto, and F. Chinesta. An error estimator for real-time simulators based on model order reduction. Advanced Modeling and Simulation in Engineering Sciences, 2(1), 2015.

[2] P.-E. Allier, L. Chamoin, and P. Ladevèze. Proper Generalized Decomposition computational methods on a benchmark problem: introducing a new strategy based on Constitutive Relation Error minimization. Advanced Modeling and Simulation in Engineering Sciences, 2(1):17, dec 2015.

[3] J. P. M. Almeida. A basis for bounding the errors of proper generalised decomposition solutions in solid mechanics. International Journal for Numerical Methods in Engineering, 94(10):961-984, jun 2013.

[4] J. P. M. Almeida and E. A. Maunder. Equilibrium Finite Element Formulations. John Wiley \& Sons, Ltd, Chichester, UK, feb 2017.

[5] J. P. M. Almeida and O. J. Pereira. Upper bounds of the error in local quantities using equilibrated and compatible finite element solutions for linear elastic problems. Computer Methods in Applied Mechanics and Engineering, 195(4-6):279-296, 2006.

[6] O. J. B. Almeida Pereira and J. P. M. Almeida. Dual adaptive finite element refinement for multiple local quantities in linear elastostatics. International Journal for Numerical Methods in Engineering, (February), oct 2010.

[7] E. Alpaydin. Introduction to machine learning. MIT press, 2020.

[8] A. Ammar. The proper generalized decomposition: A powerful tool for model reduction. International Journal of Material Forming, 3(2):89-102, 2010.

[9] A. Ammar, F. Chinesta, P. Diez, and A. Huerta. An error estimator for separated representations of highly multidimensional models. Computer Methods in Applied Mechanics and Engineering, 199(25-28):1872-1880, 2010.

[10] A. Ammar, E. Pruliere, J. Férec, F. Chinesta, and E. Cueto. Coupling finite elements and reduced approximation bases. European Journal of Computational Mechanics, 18(5-6):445-463, jan 2009.

[11] S. Boschert and R. Rosen. Digital Twin - The Simulation Aspect, pages 59-74. Springer International Publishing, Cham, 2016.

[12] L. Chamoin, P. E. Allier, and B. Marchand. Synergies between the constitutive relation error concept and PGD model reduction for simplified V\&V procedures. Advanced Modeling and Simulation in Engineering Sciences, 3(1), 2016.

[13] L. Chamoin and P. Ladevèze. Robust control of PGD-based numerical simulations. European Journal of Computational Mechanics, 21(3-6):195-207, dec 2012.

[14] L. Chamoin, F. Pled, P.-E. Allier, and P. Ladevèze. A posteriori error estimation and adaptive strategy for PGD model reduction applied to parametrized linear parabolic problems. Computer Methods in Applied Mechanics and Engineering, 327(2017):118-146, dec 2017.

[15] L. Chamoin and H. Thai. Certified real-time shape optimization using isogeometric analysis, PGD model reduction, and a posteriori error estimation. International Journal for Numerical Methods in Engineering, pages 1-33, 2019.

[16] F. Chinesta, E. Cueto, E. Abisset-Chavanne, J. L. Duval, and F. E. Khaldi. Virtual, Digital and Hybrid Twins: A New Paradigm in Data-Based Engineering and Engineered Data. Archives of Computational Methods in Engineering, 27(1):105-134, jan 2020.

[17] F. Chinesta and P. Ladevèze. Separated Representations and PGD-Based Model Reduction, volume 554 of CISM International Centre for Mechanical Sciences. Springer Vienna, Vienna, 2014.

[18] J. M. de Almeida and J. Reis. An efficient methodology for stress-based finite element approximations in twodimensional elasticity. International Journal for Numerical Methods in Engineering, n/a(n/a).

[19] J. Debongnie, H. Zhong, and P. Beckers. Dual analysis with general boundary conditions. Computer Methods in Applied Mechanics and Engineering, 122(1-2):183-192, apr 1995.

[20] P. Díez, S. Zlotnik, A. García-González, and A. Huerta. Encapsulated pgd algebraic toolbox operating with high-dimensional data. Archives of Computational Methods in Engineering, 2019. 
[21] E. Florentin and P. Díez. Adaptive reduced basis strategy based on goal oriented error assessment for stochastic problems. Computer Methods in Applied Mechanics and Engineering, 225-228:116-127, 2012.

[22] B. Fraeijs de Veubeke. Upper and lower bounds in matrix structural analysis. LTAS, 72:36, 011963.

[23] V. Ivannikov, C. Tiago, J. Moitinho de Almeida, and P. Díez. Meshless methods in dual analysis: Theoretical and implementation issues. Engineering Analysis with Boundary Elements, 37(12):1728-1744, dec 2013.

[24] K. Kergrene, L. Chamoin, M. Laforest, and S. Prudhomme. On a Goal-Oriented Version of the Proper Generalized Decomposition Method. Journal of Scientific Computing, feb 2019.

[25] K. Kergrene, S. Prudhomme, L. Chamoin, and M. Laforest. A new goal-oriented formulation of the finite element method. Computer Methods in Applied Mechanics and Engineering, 327:256-276, dec 2017.

[26] P. Ladevèze and L. Chamoin. On the verification of model reduction methods based on the proper generalized decomposition. Computer Methods in Applied Mechanics and Engineering, 200(23-24):2032-2047, jun 2011.

[27] D. Modesto, S. Zlotnik, and A. Huerta. Proper generalized decomposition for parameterized Helmholtz problems in heterogeneous and unbounded domains: Application to harbor agitation. Computer Methods in Applied Mechanics and Engineering, 295:127-149, 2015.

[28] W. Prager and J. L. Synge. Approximations in elasticity based on the concept of function space. Quarterly of Applied Mathematics, 5(3):241-269, oct 1947.

[29] J. Reis, J. P. Moitinho de Almeida, P. Díez, and S. Zlotnik. Error estimation for proper generalized decomposition solutions: A dual approach. International Journal for Numerical Methods in Engineering, n/a(n/a).

[30] R. Swischuk, L. Mainini, B. Peherstorfer, and K. Willcox. Projection-based model reduction: Formulations for physics-based machine learning. Computers and Fluids, 179:704-717, 2019.

[31] O. Zienkiewicz and J. Zhu. The three R's of engineering analysis and error estimation and adaptivity. Computer Methods in Applied Mechanics and Engineering, 82(1-3):95-113, sep 1990.

[32] S. Zlotnik, P. Díez, A. G. González, and A. Huerta. encapsulated PGD toolbox. https://git.lacan.upc.edu/zlotnik/algebraicPGDtools, 2018. 